Ebisu Ebisu

Études japonaises Études japonaises

$51 \mid 2014$

Le rapprochement franco-japonais dans l'entre-deuxguerres

\title{
Un préhistorien japonais à Paris : Nakaya Jiujirō
} (1929-1932)

先史学者・中谷治宇二郎のパリ滞在（1929-1932）

A Japanese Prehistorian in Paris: Nakaya Jiujirō (1929-1932)

\section{Laurent Nespoulous}

\section{OpenEdition}

\section{Journals}

Édition électronique

URL : http://journals.openedition.org/ebisu/1431

DOI : 10.4000/ebisu. 1431

ISSN : 2189-1893

Éditeur :

Institut français de recherche sur le Japon (UMIFRE 19 MAEE-CNRS), Maison franco-japonaise

\section{Référence électronique}

Laurent Nespoulous, « Un préhistorien japonais à Paris : Nakaya Jiujirō (1929-1932) », Ebisu [En ligne], 51 | 2014, mis en ligne le 01 novembre 2014, consulté le 19 avril 2019. URL : http:// journals.openedition.org/ebisu/1431 ; DOI : 10.4000/ebisu.1431 


\section{Un préhistorien japonais à Paris : Nakaya Jiujirō (1929-1932)}

\section{Laurent Nespoulous}

先史学者・中谷治宇二郎のパリ滞在（1929-1932）

ロラン・ネスプルス

A Japanese Prehistorian in Paris:

Nakaya Jiujirō (1929-1932)

Laurent Nespoulous

Mots-clés : archéologie, préhistoire, histoire, Nakaya Jiujirō, Marcel Mauss, abbé Breuil, Paul Rivet, Georges Henri Rivière.

L'auteur : Laurent Nespoulous est docteur en archéologie, maitre de conférences à l'Inalco (Paris), actuellement chercheur détaché à la Maison franco-japonaise (UMIFRE 19 MAEDI CNRS). Auteur de plusieurs publications sur l'histoire de l'archéologie japonaise, il travaille sur les processus de formation du pouvoir politique et de l'État au sein des sociétés de la protohistoire de l'archipel japonais.

Résumé : La figure de Nakaya Jiujirō (1902-1936), archéologue préhistorien japonais, présent à Paris de 1929 à 1932, occupe une place particulière au sein du riche contexte de l'entre-deux-guerres, tout autant qu'elle éclaire l'histoire de l'archéologie japonaise durant cette période, ainsi que celle des relations franco-japonaises. Nakaya, en raison de sa mort précoce, à l'âge de 34 ans, ne peut guère être considéré comme un des archéologues réellement déterminants dans la formation de la Préhistoire comme discipline archéologique au Japon. Cette dernière se développe surtout après la Seconde Guerre mondiale, dans un contexte intellectuel de recentrement sur l'archipel. Le présent article se propose, dans une ambition avant tout documentaire, d'évoquer la figure de ce préhistorien qui a marqué les débuts de l'archéologie préhistorique japonaise, et d'en souligner la dimension franco-japonaise éminente. 
】キーワード

先史学研究史、中谷治宇二郎、

マルセル・モース、ポール・リヴェー、ブルユ神父、

ジョルジュ = アンリー・リビエール

\section{著者}

ロラン・ネスプルスはフランス国立東洋言語 文化大学 (イナルコ) にて日本原史・古代史 准教授であり、現在フランス外務省出向研究 員として日仏会館フランス国立日本研究セン ターにて勤務中である。日本原史考古学の博 土論文の他に日本原史や日本考古学研究史な ぞを対象にした複数の論文を出している。

\section{要旨}

日本の先史学者であり、1929 年から 1932 年 にかけてパリに滞在した中谷治宇二郎 (19021936）は、両大戦間期において特別な位置を 占めるとともに、この時期の日本の考古学史 および日仏関係を知る上で重要な存在である。 34 歳で夭折した中谷は、日本においては特に 第二次大戦後に発展した先史考古学の形成に 決定的な影響を与えることはなかった。本稿 では資料に基づき、日本の先史考古学の先駆 者である中谷の足跡を追い、特に日仏両国に 関わる側面に光を当てたい。
\ Keywords: Archaeology,

Prehistory, History, Nakaya Jiujirō, Marcel Mauss, Abbé Breuil, Paul Rivet, Georges Henri Rivière.

The Author: Laurent Nespoulous is Associate Professor of Ancient Japanese History and Archaeology at Inalco (Paris) and currently assigned to the French Research Institute on Japan in Tokyo (UMIFRE 19 MAEDI CNRS). He is the author of a PhD dissertation and various articles in French and Japanese on Protohistory and the history of Japanese archaeology.
Abstract: The Japanese archaeologist Nakaya Jiujirō (1902-1936), who lived in Paris from 1929 to 1932, holds a special place in the context of the interwar years, shedding light on the history of Japanese archaeology and French-Japanese scientific interactions during this period. Having met an untimely death at the age of 34 , Nakaya cannot be considered a key figure in the genesis of prehistoric archaeology in Japan. From a disciplinary point of view, prehistory reached maturity after the Pacific War as Japan became increasingly defined by its insularity. The present contribution aims, in a documentary perspective, to present the life and work of a landmark figure in Japan's fledgling prehistoric archaeology and underline its FrenchJapanese dimension. 


\title{
Un préhistorien japonais à Paris Nakaya Jiujirō (1929-1932)
}

\author{
Laurent NESPOULOUS*
}

La figure de Nakaya Jiujirō 中谷治宇二郎 (1902-1936), archéologue préhistorien japonais, présent à Paris de 1929 à 1932, occupe une place particulière au sein du riche contexte de l'entre-deux-guerres, tout autant qu'elle éclaire l'histoire de l'archéologie japonaise durant cette période, ainsi que celle des relations franco-japonaises. Le présent article se proposera donc, dans une ambition avant tout documentaire, d'évoquer le parcours de ce préhistorien qui a marqué les débuts de l'archéologie préhistorique japonaise, et d'en souligner la dimension franco-japonaise.

La place particulière de Nakaya peut largement être imputée à deux facteurs : d'une part, nous verrons qu'il est original dans l'entreprise archéologique qui est la sienne; d'autre part, son décès précoce à l'âge de 34 ans le privera de postérité. Il convient donc de commencer par se demander en quoi, aujourd'hui, Nakaya Jiujirō peut contribuer à éclairer l'histoire de la discipline archéologique au Japon. Voici une question en effet légitime, à laquelle il nous semble possible de réagir de plusieurs façons afin d'éclairer le modeste objectif du présent article.

Tout d'abord, nous pouvons arguer que se pencher sur les aspects de la formation d'une discipline, ici la Préhistoire au Japon, en se contentant de ne prendre en compte que ses figures couronnées de succès constituerait une vision étroite du développement de l'esprit scientifique. La science

* Inalco, MFJ UMIFRE 19 (MAEDI/CNRS). 
n'est ni exclusivement faite d'" histoires à succès ", ni de généalogies de discours ininterrompues. Évoquer des personnages dont l'aventure intellectuelle - même fort brève - s'est arrêtée avec leur propre vie permet, loin de brouiller une vision de la marche de la science, de prendre conscience de la pluralité qui constitue le contexte de naissance de certaines préoccupations scientifiques ${ }^{1}$.

En outre, il se trouve que l'entre-deux-guerres constitue pour l'archéologie au Japon, mais également en France, une période de changements. En France, l'archéologie va plus que jamais se caractériser par son rayonnement en Préhistoire (Coye 2000 ; Coye \& Hurel 2011), à telle enseigne que la Protohistoire qui, de Gabriel de Mortillet ${ }^{2}$ à Joseph Déchelette ${ }^{3}$, fut fort dynamique, tend à devenir quantité négligeable et se cantonne aux "Antiquités nationales » (Lehoërff 2009). En France, la Préhistoire l'emporte sur la Protohistoire, et l'archéologie classique, centrée sur le monde méditerranéen et ses influences, demeure la plus vigoureuse de toutes. Avec la mort de Déchelette, en 1914, la Protohistoire connaîtra peu d'avancées fondamentales en France jusqu'à l'après-guerre, à la différence des pays européens voisins ${ }^{4}$.

Durant l'époque d'Edo (1603-1868), on collectionne et on classe les vestiges du passé. La poterie, notamment, fait l'objet de beaucoup d'attention et c'est ainsi que l'on découvre la poterie Jōmon, qui est alors désignée du terme de poterie de Kamegaoka (Kamegaoka doki 亀ヶ岡土器), d'après le gisement éponyme situé dans le nord-est de l'archipel. C'est dans ce cadre également que l'on va partir à la recherche des tertres impériaux consignés dans les textes classiques, eux aussi largement redécouverts par les lettrés de l'Ancien Régime japonais. Sans pouvoir parler d'archéologie, cet intérêt pour les choses anciennes constitue une des conditions qui permettront à cette discipline de se développer, plus tard, à l'ère Meiji ${ }^{5}$.

1. Nakaya fait ainsi partie de ces figures comme Jules Reboux, en France, préhistorien amateur sans postérité mais innovateur en son temps (Schlanger 2013).

2. Voir l'ouvrage Le Préhistorique. Antiquité de l'homme (de Mortillet 1885).

3. Voir le monumental manuel publié en 4 volumes (Déchelette 1908-1914).

4. Sur les différents courants de l'archéologie en Europe aux $\mathrm{XVIII}^{\mathrm{e}}$ et $\mathrm{XIX}^{\mathrm{e}}$ siècles, voir l'article d'Alain Schnapp (1982).

5. Sur le Japon d'avant l'ère Meiji, voir les contributions de Laurent Nespoulous (2003, 2012). 
L'archéologie scientifique verra le jour, à la fin du XIxe siècle, autour de l'université impériale de Tokyo, pour être ensuite conduite sous l'impulsion de la Société d'anthropologie de Tokyo (Tōkyō jinrui gakkai 東京人類學會), créée en 1884, puis du laboratoire d'anthropologie de cette même université, fondé en 1892 et dirigé par l'anthropologue Tsuboi Shōgorō

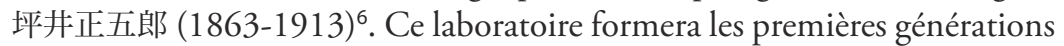
d'archéologues japonais et Nakaya en est justement issu, comme on le verra plus bas. S’y ajoute la Société d'archéologie (Nihon kōko gakkai 日本考 古學會), fondée en 1895 à Tokyo par les chercheurs du Musée impérial de Tokyo (Tōkyō teishitsu hakubutsukan 東京帝室博物館), autour notamment de l'historien Takahashi Kenji 高橋健自 (1871-1929) .

L'archéologie japonaise connaîtra ensuite de grandes avancées, en particulier sur le plan des méthodes de classification des objets et de l'analyse des sites, sous l'impulsion du laboratoire d'archéologie de l'université impériale de Kyoto, fondé en 1916. Hamada Kōsaku 濱田耕作 (1881-1938), qui en assure la direction à ses débuts, traduit en 1932 les travaux du Suédois Oscar Montelius sur la méthode de typologie et de sériation, qu’il enseigne déjà dans ses cours.

Les années 1920 constituent l'époque des grands choix et des expérimentations méthodologiques de l'archéologie japonaise, afin de déterminer les temps d'avant l'histoire au Japon. L'adoption des méthodes de l'archéologie scandinave pose les bases de ce que sera la Protohistoire au Japon. C'est dans ce contexte que Nakaya s'emploie à donner une première forme rigoureuse à un « âge de la pierre » du Japon (sekki jidai 石器時代). Il se rend alors en France en 1929 afin de préciser ce que doit être, autant sur le fond que dans ses méthodes, la Préhistoire japonaise.

L'entre-deux-guerres est donc un moment important puisqu'il coïncide à la fois avec un fort rayonnement de la France en Préhistoire, une réelle volonté de mise en ordre des temps d'avant l'Antiquité au Japon, et une grande " mobilité universitaire ». C'est véritablement une période unique d'internationalisation des champs intellectuels (Sapiro 2009 : 111-146).

6. Ce laboratoire n'a cependant pas le statut de section, qu'il n'obtiendra qu'en 1938.

7. Sur les moments de l'institutionnalisation de l'archéologie japonaise, à la fin du $\mathrm{XIX}^{\mathrm{e}}$ siècle et au début du $\mathrm{Xx}^{\mathrm{e}}$ siècle, voir les contributions de Laurent Nespoulous (2004) et d'Arnaud Nanta (2004a : 25-30). 
Des échanges dont l'intensité sera brisée par le déclenchement de la guerre en Europe, en 1939.

Ainsi, Nakaya est représentatif d'un moment de l'élaboration de l'archéologie japonaise. En outre, son ouverture à la Préhistoire telle qu'elle est pratiquée en France, à la fin des années 1920, permet, d'une part, d'appréhender les ambitions scientifiques d'un préhistorien japonais avant même que la Préhistoire ne soit une discipline au Japon et, d'autre part, de saisir dans ce regard japonais une image de la Préhistoire française et le sens qu'elle recouvre dans le discours d'un chercheur extérieur au monde européen.

\section{Nakaya Jiujirō (1902-1936)}

Nakaya, dans son parcours, pourra paraître comme une figure attachante. Pour autant, il ne s'agit en aucun cas de faire de la présente contribution une entreprise hagiographique, déjà fort bien menée du reste : plusieurs ouvrages, dont un à l'occasion du cinquantenaire de son décès, permettent de se faire une bonne idée de l'homme qu'il était. On pourra noter parmi eux le très précieux carnet, publié en 1985, portant sur la période de son séjour à Paris, dont nous avons pu, malgré ses imprécisions, tirer grand profit (Nakaya 1985).

Jiujirō est le cadet de Nakaya Ukichirō 中谷宇吉郎 (1900-1962), physicien et écrivain, qui demeure le plus connu de ces deux frères Nakaya. Ukichirō rapporte ${ }^{8}$ que Jiujirō, encore collégien, fut l'auteur d'une nouvelle qui aurait impressionné Akutagawa Ryūnosuke, au point de pousser ce dernier à rédiger un court texte intitulé Hitori no mumei sakka 一人の無名作家 (Un écrivain inconnu) ${ }^{9}$.

Né en 1902, Nakaya Jiujirō entre à l'université Tōyō, en 1922, où il entreprend des études sur la pensée indienne. Il arrête pour raison de santé presque immédiatement. En 1924, il entre à la faculté des sciences de

8. Court texte publié dans le quotidien Nishi nippon shinbun 西日本新聞, le 18 juillet 1955, disponible dans le recueil de textes de Nakaya Ukichirō (2011).

9. Le texte d'Akutagawa auquel fait référence Ukichirō est publié dans le quatrième volume de l'anthologie des œuvres d'Akutagawa des éditions Chikuma (1971). 


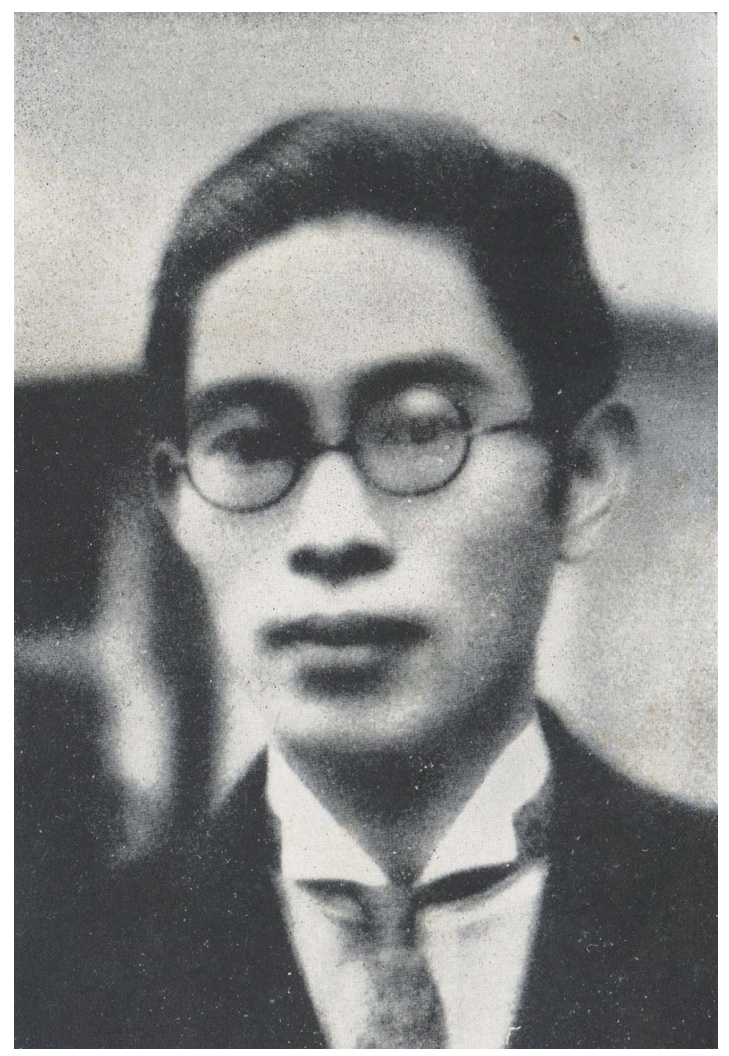

Nakaya Jiujirō, photo tirée de la réédition de son précis de Préhistoire (Nakaya 1943 [1929] : 1) 
l'université impériale de Tokyo, où il rejoint le Laboratoire d'anthropologie $^{10}$. Après le décès de Tsuboi en 1913, le laboratoire est sous la direction informelle de l'anthropologue et archéologue Torii Ryūzō 鳥居龍藏 (18701953), notamment actif en Corée coloniale aux côtés des archéologues de l'université impériale de Kyoto. Le laboratoire passe ensuite, en 1923, sous la direction effective de l'anthropologue physique Matsumura Akira 松村膫 (1880-1936), après une querelle de pouvoir entre ce dernier et Torii. Matsumura resta fidèle aux grandes directions impulsées par Tsuboi, son professeur, et contribua à présenter au Japon les découvertes paléolithiques faites en Chine autour de 1930. Ainsi le laboratoire continua-t-il à former autant d'archéologues que d'anthropologues dans les décennies 1920 et 1930 ${ }^{11}$. Nakaya en sort en 1927 et travaillera alors essentiellement sur la Préhistoire du nord-est de l'archipel, sur laquelle il publiera articles et ouvrages. Fruit de sa recherche à l'université impériale de Tokyo, sa première publication d'importance, en 1927, est une étude typologique et spatiale sur la poterie de type $c h \bar{u} k \bar{o}$ 注口12 (Nakaya 1927).

\section{Le temps d'un amateurisme fécond}

Les promesses brisées par la mort précoce de Nakaya sont à rapprocher du destin de son camarade Morimoto Rokuji 森本六爾 (1903-1936), rentré lui aussi en piètre condition physique de France, et qu'il nous faut présenter ici. Il arrive à Paris au printemps de l'année 1931, avec pour première intention de poursuivre son chemin vers Londres. Tout comme Nakaya depuis 1929, Morimoto réside finalement une année à la Maison du Japon (ouverte en 1927) de la Cité universitaire internationale. À vrai dire, Morimoto aurait sans doute constitué un sujet plus pertinent, d'un

10. Dans ses articles en français, Nakaya signe en indiquant qu'il est « membre de l'Institut d'anthropologie de l'Université impériale de Tokio ", désignation qui renvoie en réalité au laboratoire.

11. Sur les organismes, revues et chercheurs du monde archéologique de la capitale durant cette période, voir le travail d'Arnaud Nanta (2004b : 418-427). Le laboratoire d'anthropologie s'orientera cependant vers l'anthropologie physique, outre la paléoanthropologie, après 1938, soit après le décès de Matsumura.

12. Poterie à bec verseur, considérée de nos jours comme appartenant au Jōmon récent et final, du I $^{\mathrm{e}}$ millénaire au début du $\mathrm{I}^{\mathrm{er}}$ millénaire avant notre ère. 
point de vue scientifique, que celui de Nakaya, car sa production académique au Japon fut très abondante, consistant elle aussi à ordonner le Japon d'avant l'histoire. Nous pouvons ainsi évoquer, publié en 1929, son important travail intitulé Nihon seidōki jidai chimei-hyō 日本青銅器時代 地名表, «Inventaires des gisements de l'âge du bronze au Japon » (Morimoto 1929). Nakaya publie la même année, chez le même éditeur Oka shoin 岡書院, ce qui est sans doute le premier « manuel » de Préhistoire du Japon, intitulé, lui aussi selon la division chronologique scandinave, Nihon sekki jidai teiyo 日本石器時代提要, “Précis sur l'âge de la pierre au Japon» (Nakaya 1929). Morimoto est un personnage important, force motrice dans la fondation de la Société d'études archéologiques (Kōkogaku kenkyū-kai 考古學 研究會), en 1927 (renommée Société d'archéologie de Tokyo, Tōkyō Kōkogaku-kai 東京考古學會, en 1929), et de sa revue, lancée en 1929, Kökogaku kenkyū 考古學研究 (La Recherche archéologique), qui devient Kökogaku 考古學 (L'Archéologie) peu de temps après, et dont il sera le rédacteur en chef. Il marque l'histoire de l'archéologie de l'archipel notamment parce que, dans un ouvrage édité par la Société d'archéologie de Tokyo - recueil de textes cette fois-ci -, il est, en 1933, le premier archéologue au Japon à définir la culture Yayoi comme celle des premières sociétés agraires (rizicultrices, en l'occurrence) de l'archipel ${ }^{13}$, idée qui sera largement reprise après-guerre et fortement développée dans les années 1960 et $1970^{14}$.

Comme Nakaya, il n'est pas titulaire d'un poste dans une institution de recherche de l'époque et est donc, faute de meilleur qualificatif, un " amateur », comme le furent beaucoup d'archéologues de cette époque. Il

13. Cette première tentative de définition des premières sociétés agraires de l'archipel s'intitule Nihon genshi nōgyō 日本原始農業 (Une agriculture primitive au Japon) (Morimoto 1933), dix ans après que V. G. Childe ait lancé pour la première fois l'idée de " révolution néolithique " pour marquer la formation des premières sociétés agraires, dans son ouvrage How Labour Governs (Childe 1923). Deux ans plus tard, Childe est l'auteur d'un nouvel ouvrage dans lequel il brosse un tableau européen de la "néolithisation " depuis le Proche-Orient, The Dawn of European Civilization (Childe 1925) et qui connaîtra de nombreuses rééditions.

14. Le thème de l'agriculture dans les temps anciens au Japon est un sujet récurrent de l'archéologie depuis l'ère Meiji. Morimoto apporte une dimension nouvelle en abordant autant la question par le volet technologique que, et c'est cela qui constitue une nouveauté, par le type de société impliquée. Sur ce thème fondateur de la discipline archéologique au Japon, voir la présentation qu’en fait Kobayashi Yukio (1971). 

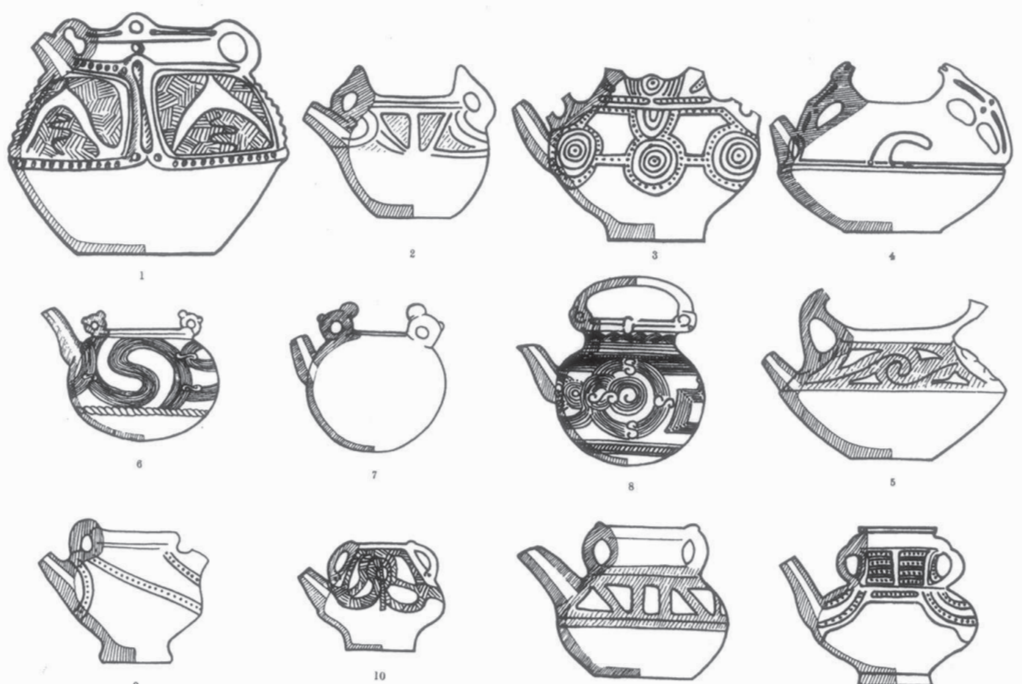

Type A
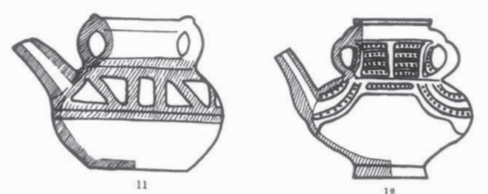

$10 \mathrm{~cm}$
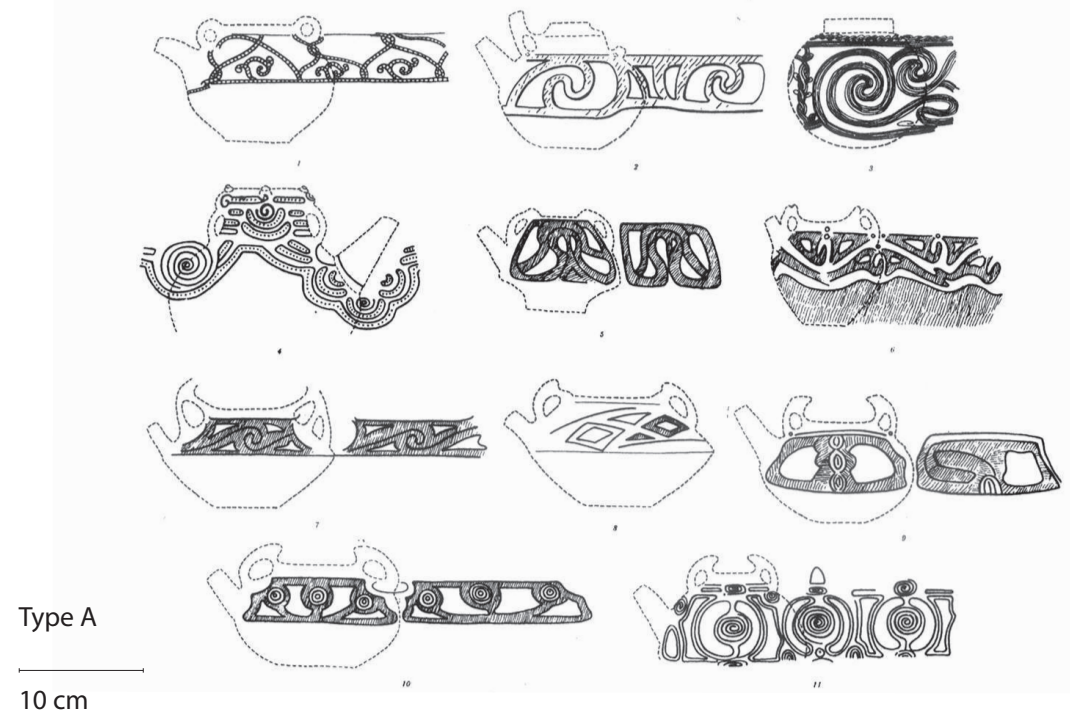

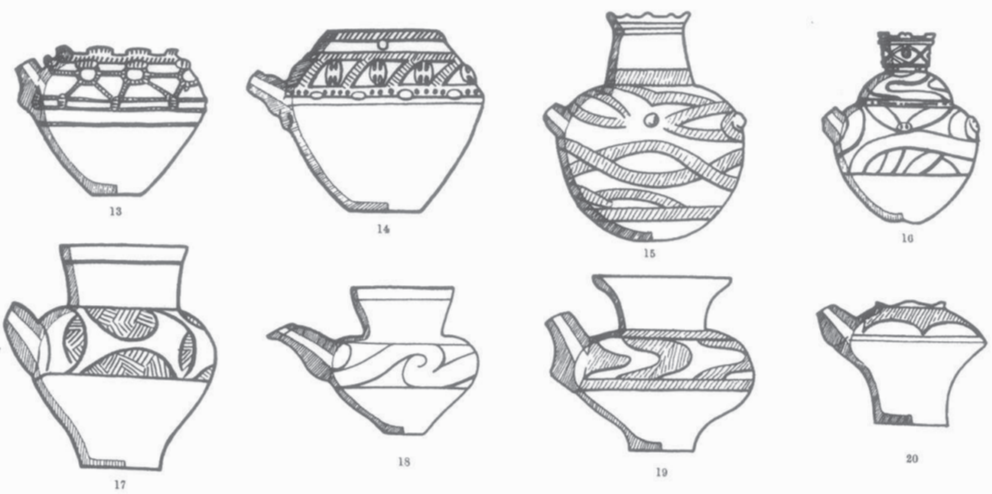

18
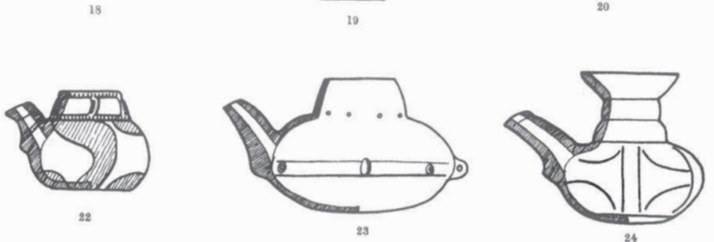

Type B

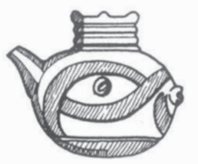

22

$10 \mathrm{~cm}$
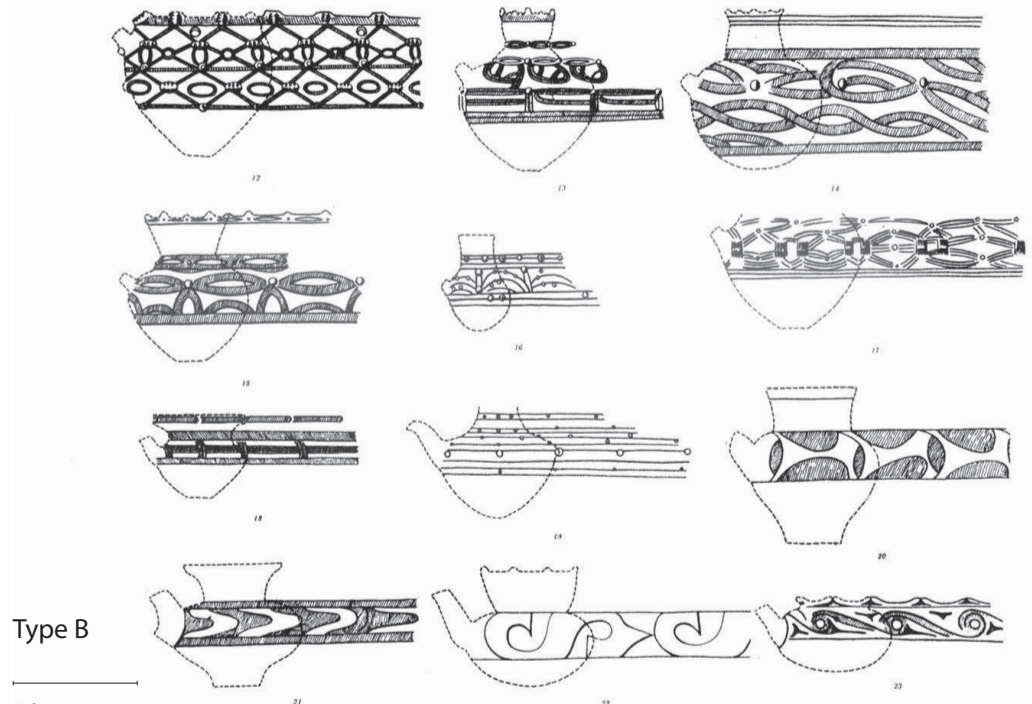

$10 \mathrm{~cm}$

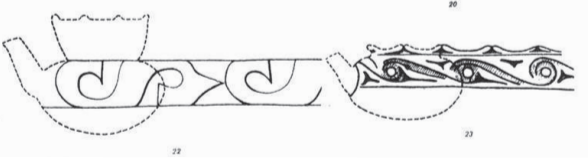




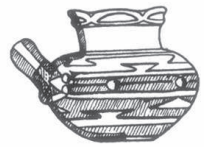

25

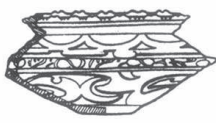

20

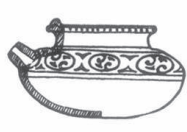

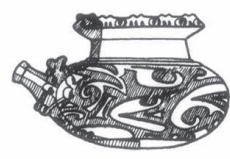

27

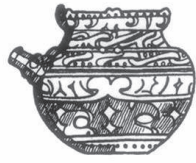

28

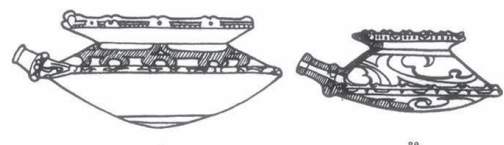

3

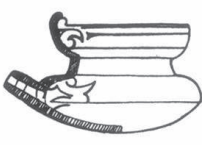

35

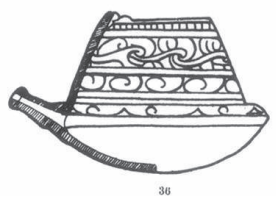

Type C

$10 \mathrm{~cm}$
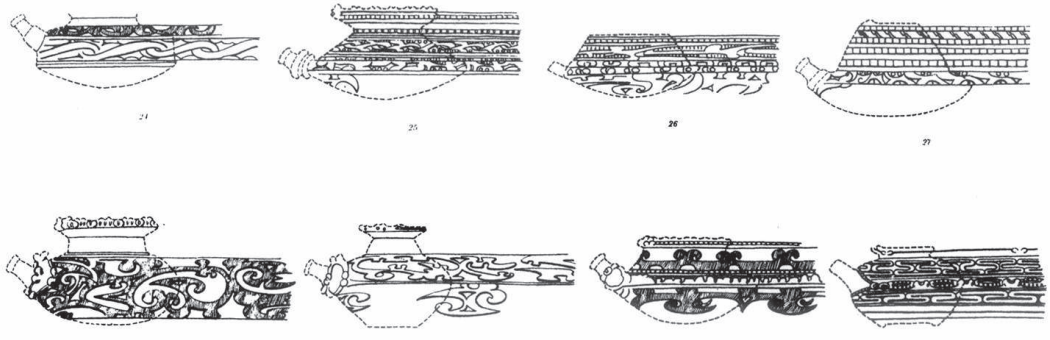

(5)
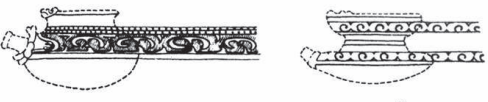

$\sqrt{2 \quad 20}$

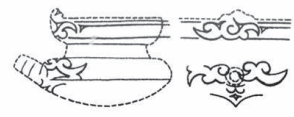

Type C
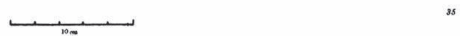

$10 \mathrm{~cm}$ 


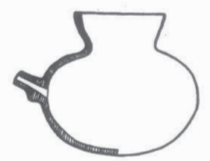

37

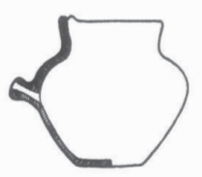

43

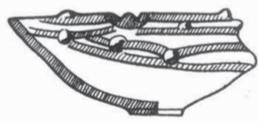

45
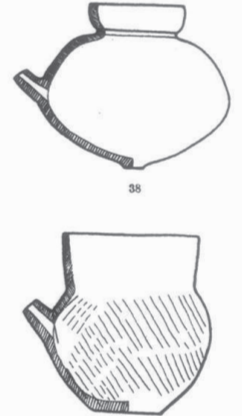

44

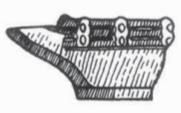

40

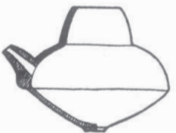

39

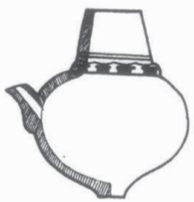

42

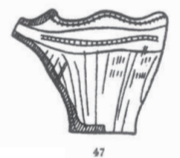

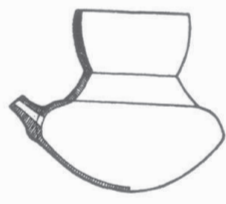

40
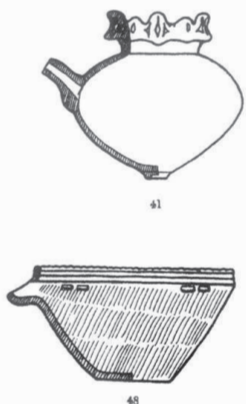

Type D

$10 \mathrm{~cm}$

Double pages précédente et présente Typologie des poteries à bec (types $A, B, C$ et $D$ ) réalisée par Nakaya sur la base de la forme et du décor (Nakaya 1927 : 155-158) 


\begin{tabular}{|c|c|c|c|c|c|}
\hline 順位 & 國 & 別 & 遺跡數 & 注口土器 & 平 均 \\
\hline 1 & 樾 & 前 & 73 & 38 & 0.52 \\
\hline 2 & 陵 & 奧 & 240 & 87 & 0.36 \\
\hline 3 & 狗 & 挠 & 410 & 61 & 0.15 \\
\hline 4 & 常 & 陵 & 292 & 42 & 0.14 \\
\hline 5 & 下 & 總 & 275 & 22 & 0.08 \\
\hline 6 & 磐 & 城 & 81 & 6 & 0.07 \\
\hline 7 & 㹂 & 中 & 210 & 14 & 0.07 \\
\hline 8 & 武 & 藏 & 826 & 7 & 0.01 \\
\hline
\end{tabular}

a) Classement régional de densité en poteries à bec (poteries à bec/ nb total sites)

\begin{tabular}{|c|c|c|c|c|c|}
\hline 順位 & 國 & 別 & 造䟢數 & 土假數 & 平 均 \\
\hline 1 & 煺 & 前 & 73 & 28 & 0.38 \\
\hline 2 & 常 & 陸 & 292 & 102 & 0.35 \\
\hline 3 & 陸 & 奥 & 240 & 85 & 0.35 \\
\hline 4 & 下 & 總 & 275 & 73 & 0.27 \\
\hline 5 & 社 & 後 & 410 & 53 & 0.13 \\
\hline 6 & 旣 & 城 & 81 & 4 & 0.05 \\
\hline 7 & 陸 & 中 & 210 & 11 & 0.05 \\
\hline 8 & 武 & 㶓 & 826 & 32 & 0.04 \\
\hline
\end{tabular}

c) Classement régional de densité en figurines (figurines/nb total sites)

\begin{tabular}{|c|c|c|c|c|c|}
\hline 順位 & 國 & 別 & 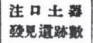 & 注口上 & 平均数 \\
\hline 1 & 常 & 陸 & 3 & 42 & 14.00 \\
\hline 2 & 犲 & 挠 & 10 & 61 & 6.10 \\
\hline 3 & 磐 & 㳦 & 1 & 6 & 6.00 \\
\hline 4 & 陸 & 前 & 8 & 38 & 4.75 \\
\hline 5 & 陸 & 奥 & 22 & 87 & 3.95 \\
\hline 6 & 下 & 總 & 12 & 22 & 1.83 \\
\hline 7 & 陵 & 中 & 9 & 14 & 1.55 \\
\hline 8 & 武 & 藏 & 6 & 8 & 1.33 \\
\hline
\end{tabular}

b) Classement régional de densité en poteries à bec (poteries à bec/ gisements de poteries à bec)

\begin{tabular}{|c|c|c|c|c|c|}
\hline 順位 & 國 & 䟝 & 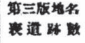 & 席石爷数 & 乐均 \\
\hline 1 & 常 & 选 & 115 & 53 & 0.46 \\
\hline 2 & 陵 & 前 & 46 & 21 & 0.45 \\
\hline 3 & 下 & 總 & 166 & 64 & 0.39 \\
\hline 4 & 陸 & 奧 & 192 & 44 & 0.23 \\
\hline 5 & 磐 & 城 & 69 & 10 & 0.15 \\
\hline 6 & 阹 & 中 & 120 & 18 & 0.15 \\
\hline 7 & 武 & 藏 & 683 & 68 & 0.10 \\
\hline 8 & 犲 & 後 & 383 & 13 & 0.03 \\
\hline
\end{tabular}

d) Classement régional de densité en haches polies (haches polies/ nb total sites)
Graphique

Corrélation a, c et d

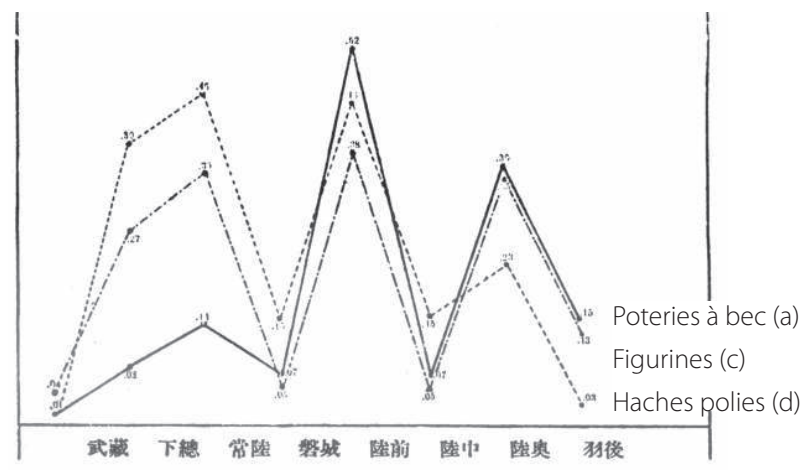




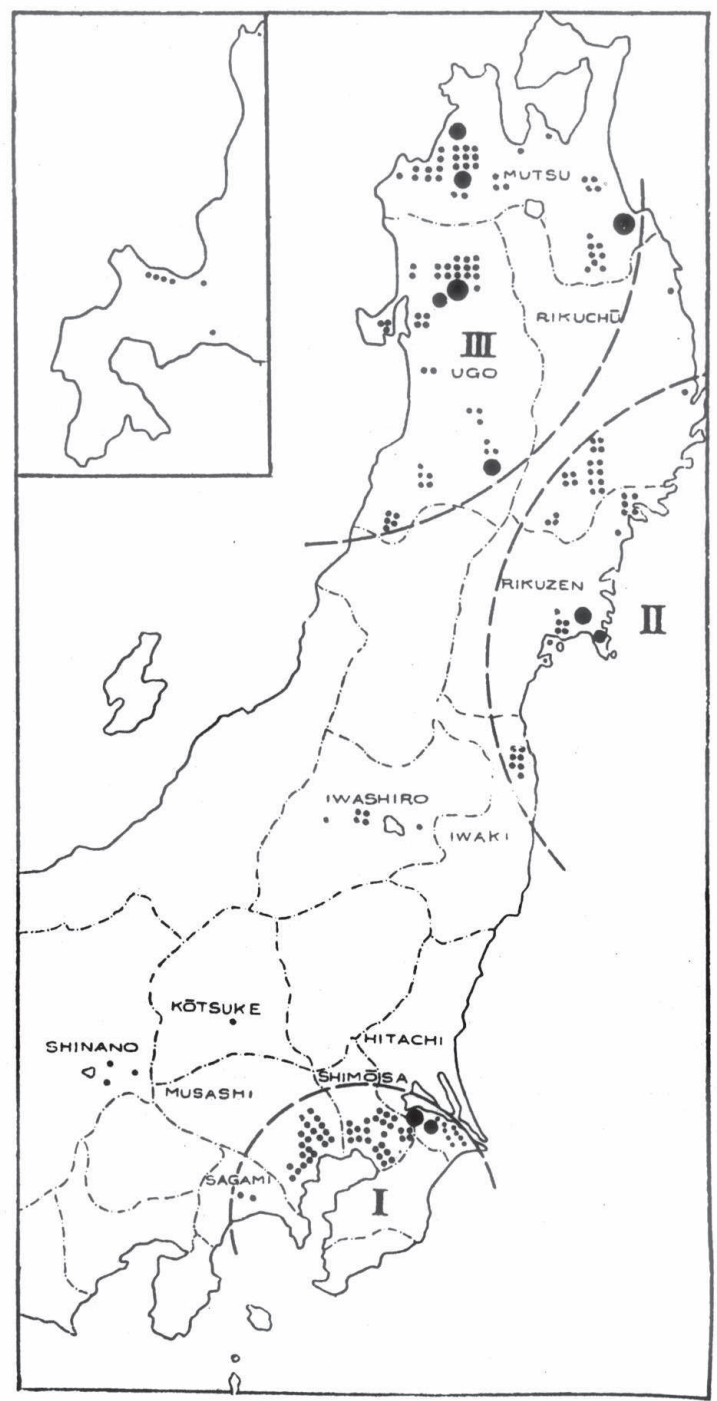

Distribution régionale de la poterie à bec 
fera néanmoins des émules dans le système académique. Kobayashi Yukio 小林行雄 (1911-1989), de l'université impériale de Kyoto, archéologue incontournable de l'après-guerre, est un de ses " disciples " spirituels. L'empreinte de Morimoto au Japon est donc incomparablement plus forte que celle de Nakaya. Toutefois, il est très difficile de se rendre vraiment compte de son activité en France, alors que celle de Nakaya laisse une trace relativement aisée à suivre, particulièrement de par son activité au sein de la Société préhistorique française, ainsi que le carnet de son séjour, publié en 1985. Cette activité de Nakaya est précieuse et appréciée à Paris, car il sera le vecteur principal de la connaissance de la Préhistoire de l'archipel à cette époque en France. Nakaya, comme Morimoto, sont des amateurs, dans le sens où ils ne vivent pas de leur occupation d'archéologue. Ils consentent à investir leurs ressources personnelles afin de mener à bien la tâche scientifique qu'ils se sont fixée. À en juger par leur production en langue japonaise, nous pouvons dire qu'ils poursuivaient de manière complémentaire un objectif proche de celui accompli par un Joseph Déchelette dans son imposant Manuel d'archéologie préhistorique et celtique, terminé en 1914 (Déchelette 1908-1914).

\section{"L'âge de la pierre » : les défis d'une mise en ordre}

Nakaya fait partie des premiers archéologues japonais à tenter de mettre de l'ordre dans ce que l'on appelle de nos jours la période Jōmon. En 1928, comme le fera Morimoto un an plus tard pour l'" âge du bronze " au Japon, Nakaya dirige, pour l'université impériale de Tokyo, la publication d'un inventaire des gisements archéologiques japonais de l' « âge de la pierre ${ }^{15}$. Son précis de Préhistoire de 1929 sera suivi, en 1930, après son départ pour la France, d'une bibliographie des sources relatives à l'âge de la pierre au Japon ${ }^{16}$ (Nakaya 1929, 1930g).

15. L’ouvrage s'intitule Nihon sekki jidai ibutsu hakken chimeihyō 日本石器時代遺物發見 地名表 (Inventaire des gisements archéologiques de l'âge de la pierre au Japon), et a une vocation quasi exhaustive (Tōkyō teikoku daigaku 1928).

16. Dans cet ouvrage, Nakaya remonte aussi loin en arrière dans le temps afin d'évaluer toute la documentation susceptible d'exister, de celle rapportée par les " antiquaristes » sous l'Ancien Régime aux ramassages de l'ère Meiji. 
Dans son ouvrage de 1929, Nakaya expose dans le détail ce qui constitue les bases d'une discipline archéologique préhistorique et, ce faisant, il tente de cerner autant les questions pratiques posées par l'archéologie de terrain que les problèmes soulevés par l'organisation ultérieure des données collectées. L'ouvrage se compose ainsi de trois parties.

La première, une introduction, aborde en deux chapitres les gisements archéologiques et les artefacts d'une part, et les méthodes d'analyse et d'enregistrement d'autre part. La visée est d'évidence pédagogique, et Nakaya initie le lecteur à la pratique archéologique depuis l'étape de la localisation d'un gisement à celles des préparatifs du départ sur le terrain (quel outillage emporter) et du déroulement de la fouille. Quant aux méthodes d'analyse expliquées par Nakaya, elles sont au nombre de cinq, copieusement commentées, mettant en lumière leur utilité et leur limite : la " méthode stratigraphique " (söiteki kenkyūhō 層位的研究法), qui " guide la fouille » (Nakaya 1943 [1929] : 45-49) ; la " méthode typologique "(keitaiteki kenkyūhō 形態的研究法), qui guide la compréhension de la constitution des artefacts (p. 49-59) ; la "méthode générale " (sōgöteki kenkyūhō 綜合的研究 法), qui consiste à mettre en relation les différents gisements archéologiques afin de déterminer à la fois spatialement et chronologiquement la présence des « cultures" archéologiques (bunka 文化) (p. 59-60) ; la " méthode ethnographique " (dozoku kenkyūhō 土俗研究法) qui, par l'étude des productions de «l'homme primitif» (mikaijin 未開人), permet d'inférer le sens des artefacts des sociétés de l'âge de la pierre (p. 61-64); enfin, la « méthode psychologique " (shinrigakuteki kenkyūhō 心理學的研究法), visant à comprendre la mentalité et les intentions des hommes préhistoriques par leurs objets, et dont Nakaya recommande de se tenir à distance en raison de l'impossibilité de questionner directement les sujets (p. 64).

Nous reviendrons dans la partie suivante sur la question de la méthode spatiale, qui constitue selon nous une caractéristique de cette archéologie développée autour du laboratoire d'anthropologie de l'université impériale de Tokyo : Matsumura, son directeur, avait soutenu en 1924 une thèse de doctorat en anthropologie physique exposant la répartition spatiale des types morphologiques des Japonais. On peut comprendre toute son importance méthodologique chez Nakaya, une fois la même méthode appliquée à la construction de "cultures archéologiques » dans l'archipel japonais.

L'exposé de 1929 de Nakaya se conclut sur une définition de la Préhistoire et de son rôle, notamment par rapport à la discipline historique, ce qui 
permet non seulement de comprendre ses orientations ultérieures à Paris, mais constitue en outre une des rares définitions épistémologiques précises de son époque. Nakaya considère que l'archéologie, en tant qu'étude portant sur les vestiges matériels de l'humanité, s'inscrit à l'extrémité d'une très longue histoire des savoirs (p. 76). Cette archéologie, de plus, est envisagée en deux grands champs : celui de l' " archéologie historique » et celui de l'" archéologie préhistorique" (p. 77). La première étudie les productions des périodes historiques ou tente d'approcher par celles-ci les phénomènes culturels des sociétés concernées. La dernière entreprend de saisir les cultures d'avant l'écrit par les objets qu'elles ont laissés.

Nakaya dénonce en la première, du fait de l'état de la discipline historique au Japon, une tendance psychologisante ${ }^{17}$, qui conduit à une étude des objets comme des "productions " d'une société, sans laisser se développer librement une véritable « histoire des cultures ». Là où l'archéologie des époques historiques est inféodées aux tendances lourdes des études historiques elles-mêmes, et en partage certaines méthodes, la Préhistoire repose essentiellement sur ses méthodes d'extraction des données, la méthode stratigraphique en constituant l'élément le plus marquant (p. 78). Pour Nakaya, si une définition définitive manque encore à la Préhistoire, particulièrement celle de l'archipel, il est certain qu'elle est bien plus proche de l'ethnographie que de l'histoire (p. 80).

La deuxième partie de l'ouvrage de 1929 se compose de dix chapitres et constitue un fort dossier technique faisant le tour des matériaux de l'archéologie préhistorique. Ce dossier s'ouvre sur trois premiers chapitres consacrés aux sites archéologiques : qu'est-ce qu'un site archéologique et combien en sont comptabilisables au Japon ? (chap. I, p. 85-106) ; quels sont les types de gisements connus au Japon (chap. II et III, p. 107-166). C'est ensuite au tour des matériaux archéologiques retrouvés au Japon : qu'est-ce qu'un artefact ? (chap. Iv, p. 167-186) ; quels sont les « restes naturels» - shizenteki ibutsu 自然的遺物 (ossements humains et la question de leur race

17. 歴史學は今日のところ心理學を力學とした文化史への途を歩まうとしてるるかに見える。 少なくとも精神文化を主としない文化史は有史時代にあつては許されないことは明かである。 (L'histoire de nos jours me semble être engagée sur la voie d'une histoire culturelle ayant pour moteur la psychologie. À tout le moins, il est clair qu'une histoire culturelle qui n'aurait pas pour objet l'histoire des mentalités n'a pas droit de cité pour les époques historiques.) (Nakaya 1943 [1929] : 77). 
d'appartenance, restes végétaux et de faune, etc. - (chap. V et vI, p. 187240) ; quels sont les matériaux produits de main d'homme ? (outillage, poterie, motifs, figurines, parure, etc.) (chap. vir à X, p. 241-420).

La troisième partie de l'ouvrage (p. 421-531) correspond à une préoccupation qui sera permanente chez Nakaya : celles des sources dans lesquelles aller rechercher des traces de découvertes passées relatives à la Préhistoire, depuis l'époque d'Edo jusqu'à l'ère Meiji. Cette partie fait ainsi, en quelque sorte, office de prélude à l'ouvrage que publiera Nakaya, juste avant son décès à son retour de France, "Histoire préliminaire à la recherche en Préhistoire au Japon» (Nihon senshigaku joshi 日本先史學序史), publié en 1935 (Nakaya 1935).

Le travail de Nakaya se caractérise par deux aspects alors encore relativement rares au sein des études préhistoriques au Japon. Il est ainsi le premier chercheur à tenter une typo-chronologie détaillée de la poterie Jōmon, à laquelle il joint une nécessaire approche spatiale : tous ses travaux depuis 1927 sont copieusement enrichis de cartes de répartition. L'archéologie japonaise d'après-guerre ne retiendra pas ses conclusions chronologiques, mais il est important de noter que Nakaya est le premier à avoir proposé une approche chronologique globale de la poterie préhistorique de l'archipel.

Quand se posera, dans les années quarante, la question de publier un nouvel ouvrage sur la Préhistoire japonaise, Umehara Sueji 梅原末治 (18931983), de l'université impériale de Kyoto, se lancera dans une entreprise de réédition de l'ouvrage de Nakaya de 1929, publié en 1943 dans une version légèrement revue ${ }^{18}$ (Nakaya 1943 [1929]). Nous sommes près de dix ans après la mort de son auteur original, et quatorze ans après la publication de l'ouvrage dans sa première édition. Cette réédition laisse saisir un champ encore faiblement doté en personnel, comparativement à des champs qui relèvent de l'Antiquité ou de la Protohistoire, à savoir la période précédent immédiatement les temps antiques.

Enfin, le manuel de Nakaya, terminé à l'été 1929, juste avant de s'embarquer pour le transsibérien qui le mènera à Paris, permet de saisir un état de la pensée de Nakaya avant son séjour d'études de deux ans et demi en France. Il permet aussi de mettre en relation les éléments soulignés par

18. Umehara peut alors s’appuyer sur de jeunes archéologues comme Kobayashi Yukio, évoqué plus haut. 
Nakaya dans son livre avec le contenu de ses interactions dans le monde de la recherche française - telles que décrites dans son carnet de voyage -, ainsi qu'avec sa production scientifique en français.

\section{Nakaya Jiujirō à Paris}

Parti en France à 27 ans, Nakaya arrive à Paris à l'été 1929. Il y rencontre Serge Elisséef, sur lequel il a pu compter pour recevoir des conseils pratiques. Il est également en contact avec Sylvain Lévi, qui est à la tête de la Société asiatique et vient de diriger la Maison franco-japonaise pendant deux ans, de 1926 à 1928. Nakaya est probablement aussi en contact, avant de quitter le Japon, avec Charles Haguenauer, où ce dernier réside de juin 1924 à février 1932. Haguenauer qui a, dans ses Notions d'archéologie japonaise (publié en 1931 à la Maison franco-japonaise), les travaux de Nakaya Jiujirō pour source omniprésente (Haguenauer 1931). La richesse des fréquentations de Nakaya en France, rapportées par son carnet de voyage, frappe d'emblée.

\section{Dans l'entourage de Georges Henri Rivière et Paul Rivet}

Nakaya semble avoir été fort proche de Georges Henri Rivière (18971985), de cinq ans son aîné et futur fondateur, en 1937, du musée national des Arts et Traditions populaires. Rivière est alors le bras droit de Paul Rivet (1876-1958) ${ }^{19}$, qui dirige, depuis 1928, le musée d'ethnographie du Trocadéro, et dont Nakaya est l'élève. Rivet est un personnage très important, fondateur, en 1925, avec Marcel Mauss et Lucien Lévy-Bruhl, de l'Institut d'ethnologie de l'université de Paris. Le musée du Trocadéro, comme le musée de l'Homme plus tard, est rattaché au Muséum national d'histoire naturelle. Rivet ouvre les portes de trois institutions importantes de l'époque à Nakaya afin que celui-ci puisse travailler sur des collections ethnographiques : celles du musée d'ethnographie du Trocadéro - Rivière lui conseille de commencer par les collections de poteries péruviennes (Nakaya

19. Sur Paul Rivet, on pourra se reporter à la contribution de Carole Reynaud-Paligot (2001) et à l'ouvrage de Christine Laurière (2008). 
1985 : 65) - naturellement, mais également de l'Institut d'ethnologie et du laboratoire d'anthropologie du Muséum. Par Rivet, Nakaya fréquentera donc les cours de Marcel Mauss, duquel il se fait remarquer. Dans les échanges de Nakaya avec Rivière et Rivet, plusieurs "moments ", au cours de l'année 1929-1930, rapportés dans son carnet de voyage nous semblent sortir du lot.

Un premier se déroule un dimanche, au retour d'un voyage en Suède. Rivière invite Nakaya chez lui et lance la conversation sur l'ethnologie et l'ethnographie au Japon, émettant le souhait d'instaurer des échanges d'objets avec l'archipel (Nakaya 1985 : 66-67). Nakaya regrette alors l'absence d'institutions, musée ou laboratoire d'ethnographie, avec lesquelles mettre son interlocuteur français en contact, ajoutant que tout ce qu'il fut en mesure d'offrir à ce dernier, c'est la possibilité d'acheter des documents. Nakaya n'était pas venu du Japon les mains vides pour avancer dans son travail de classification. Ce qui nous amène à souligner, d'ailleurs, que certains objets des actuelles collections archéologiques japonaises du musée de l'Homme ont pour provenance répertoriée " Nakaya ». Ce n'est qu'après la Seconde Guerre mondiale que le Japon se dotera de puissantes institutions en ethnographie et la remarque de Nakaya permet de saisir qu'en 1930, le Japon est encore faiblement organisé sur ce plan. La France et l'Europe en général offrent donc un visage sans doute imposant.

Un autre de ces moments, cette fois-ci indiquant que Nakaya est à la recherche de "signes " montrant la bonne direction méthodologique à suivre dans la détermination des cultures préhistoriques, est lorsque, travaillant sur des travaux de 1928 de l'américaniste Alfred Kroeber (18761960) sur les poteries Nazcas du Pérou ${ }^{20}$, il remarque que l'anthropologue américain fait appel au même type d'approche typologique que lui dans son travail de 1927 sur les poteries à bec verseur de l'archipel. Jugeant que l'étude est incomplète dans la mesure où simplement réduite à la classification de la poterie, il entreprend de voir Rivet pour discuter de la question (Nakaya 1985 : 68-69).

Nakaya commence par demander à son directeur ce qu'il pense de "la méthode quantitative "( ra metöde kantetatefu ラ・メトーデ・カンテタテフ).

20. On peut indiquer, sur ce dernier sujet, à des fins de comparaisons de méthodes, l'ouvrage The Archaeology and Pottery of Nazca, Peru: Alfred Kroeber's 1926 Expedition (Kroeber \& Collier 1998). 
Rivet lui répond qu'elle est excellente. Nakaya alors de demander, dans ce cas, pourquoi, à l'avis du directeur du musée d'ethnographie du Trocadéro, Kroeber ne parvient pas à être concluant dans la restitution de la culture qu'il étudie. Rivet lui répond que c'est parce que l'anthropologue de l'université de Californie ne fait pas en même temps appel à « la méthode cartographique " (ra metōde karutogurafikuラ・メトーデ・カルトグラフィク). Nakaya qui avait la même lecture s'empresse de faire savoir à Rivet que dans son étude de 1927, il avait utilisé conjointement cartographie et typologie. Rivet de lui répondre avec fougue que, dès 1917, il avait déjà pu expérimenter ces deux approches à l'occasion d'une expédition en Équateur sur des poteries précolombiennes. Ce passage est important dans la mesure où il souligne que Nakaya fait, méthodologiquement parlant, partie des pionniers de l'étude typologique et spatiale, et ce pas seulement au Japon, tout en illustrant comment cette méthode a pu passer de l'anthropologie vers l'archéologie (le cas de Rivet est ici éclairant). Être en France dans une institution bien fournie en publication permet ainsi à Nakaya de tester la validité de son approche à l'aune de la recherche de son époque ${ }^{21}$.

Un troisième moment à relever est la rencontre de Nakaya avec Marcel Mauss. Sur la recommandation de Sylvain Lévi, et suivant ses camarades du Trocadéro, Nakaya commence à fréquenter les cours de Mauss. Il n'est pas précisé dans son carnet quelle fut la fréquence ni le contenu de ses échanges avec cette grande figure. Il souligne seulement à quel point les cours de Mauss - qu'il présente comme un vieil homme partiellement édenté, qui relève dans ses cours un manque cruel de documentation ethnographique en France, et faisant sans cesse référence à des auteurs allemands -, le conforte dans sa volonté d'avancer sur le chemin d'une approche ethnographique. De Mauss, il dresse la définition suivante :

Le professeur Mauss, héritier de la sociologie positiviste de Durkheim, était déjà lancé depuis longtemps dans l'étude des religions primitives mais, cette année [1930], il faisait particulièrement sensation dans le monde scientifique par son nouveau cours, à l'Institut d'ethnologie, où se côtoyaient l'ethnographie envisagée comme une science descriptive et la sociologie. (Nakaya 1985 : 70)

21. Nakaya découvre peu après un court texte de Paul Rivet : "L'étude des civilisations matérielles : ethnographie, archéologie, préhistoire» (Rivet 1929). 
Enfin, un autre moment « crucial » du récit de Nakaya est ce passage où l'on comprend que, conscient du temps limité dont il dispose en France, il " chasse » les sommités susceptibles de lui être d'un secours dans sa formation, qu'il entend visiblement être résolument pluridisciplinaire :

De retour du bal, je marchais aux côtés de Rivière dans la nuit froide.

" [Nakaya] Qui est le plus grand préhistorien français, mis à part Boule, le paléoanthropologue?

— [Rivière] Certainement l'abbé Breuil.

- [Nakaya] Je le connais. Il y a une très grande quantité de travaux publiés de sa plume. Qui pourrait venir en second?"

Rivière se tait.

" [Nakaya] J'ai déjà pu rencontrer une dizaine d'archéologues. Mais je pense maintenant poursuivre davantage vers l'ethnographie. Qui serait alors le plus grand de tous? Mis à part Mauss le sociologue?

- [Rivière] C'est certainement Rivet. "

Préoccupé par les quelques gouttes qui venaient de commencer à tomber, il leva les yeux vers le ciel et poursuivit : "Mauss est un grand personnage qui force l'admiration. "

Nous nous engouffrâmes dans les escaliers descendant dans le métro. (Nakaya $1985: 71)$

\section{Boule, Breuil et bien d'autres...}

À la fin de l'année 1929, Nakaya va rencontrer Marcellin Boule (18611942), titulaire de la chaire de Paléontologie au Museum d'histoire naturelle, et dont Nakaya estime qu'il est une des grandes figures fondatrices de la Paléontologie humaine en Europe. En effet, pour lui, Boule, dont il compare le physique à celui d'un dompteur de lion, est un des principaux acteurs à avoir fait de la recherche préhistorique une discipline axée sur le Paléolithique (Nakaya 1985 : 73). De fait, Marcellin Boule est une sommité de son époque. Il est bien connu pour avoir publié ce qui est, en son temps, l'étude la plus complète d'un squelette d'homme de Néandertal, trouvé en 1908 en Corrèze. Dans la discussion qui s'ouvre entre les deux préhistoriens, on apprend que Nakaya envisage de rester encore environ un an à Paris, et que, à la question de Boule portant sur ses motivations scientifiques, il répond vouloir étudier l'ethnographie et l'archéologie et, dans le cas de cette dernière, particulièrement le Néolithique (Nakaya 1985 : 71). Boule l'enjoint alors d'aller voir Salomon Reinach (1858-1932), 
archéologue antiquisant et grand érudit, directeur depuis 1902 du musée des Antiquités nationales (MAN), à Saint-Germain-en-Laye.

Nakaya ne garde pas trace dans son carnet publié en 1985 de sa rencontre, si elle a eu lieu, avec Reinach. Les passages concernant le musée de SaintGermain sont du reste assez vagues et il est difficile de savoir si Nakaya y a vraiment été actif. Concernant l'ethnographie, Boule lui confirme qu'au musée du Trocadéro, il est entre de bonnes mains en la personne de Rivet. Quant au Paléolithique, si la motivation lui en prend, Boule lui indique chaleureusement que les portes de son bureau lui sont grandes ouvertes. Il semble que la discussion se serait orientée sur les dernières découvertes en Chine. Nakaya ne rapporte pas le détail de cette conversation, mais on peut imaginer qu'elle a trait aux dernières découvertes autour de l'homme de Pékin. Quant au Japon, ils semblent tous deux d'accord pour dire qu'il y a peu d'espoir d'y trouver un jour des sites du Paléolithique. En effet, il est encore communément admis que l'archipel ne peut présenter de stade très ancien de présence des hominidés. Nakaya poursuit en demandant à Boule s'il estimerait utile d'étudier, en plus du Néolithique européen, le cas du Mésolithique, et si la tâche serait possible à accomplir en trois ou quatre mois. Ce à quoi Boule répond par l'affirmative (Nakaya 1985 : 72-73). La question de Nakaya n'est pas anodine, car il se pose la question du statut de la Préhistoire de l'archipel. Nous reviendrons plus loin sur ce point qui dépasse la simple question terminologique. Quoi qu'il en soit, Nakaya est ravi de sa rencontre avec le " dompteur de lion » du Museum d'histoire naturelle.

Peu de temps après Marcellin Boule, il rencontre l'abbé Henri Breuil (1877-1961) ${ }^{22}$, à l'issue de son premier cours suivi à l'Institut de paléontologie humaine. Nakaya précise qu'il a tôt fait de devenir un étudiant assidu de cet enseignement, très illustré, tenu à dix-sept heures, dans lequel il est question du Néolithique, mais aussi des problèmes de lecture des sites archéologiques et de méthodes de fouille ${ }^{23}$. On apprend dans ce que

22. L'abbé Breuil demeure une figure puissante de l'archéologie française, comme en témoignait encore l'exposition "Sur les chemins de la Préhistoire, L'abbé Breuil, du Périgord à l'Afrique du Sud ", qui s'est tenue au Centre d'art Jacques Henri Lartigue, du 7 mai au 17 septembre 2006. Sur Breuil, on pourra se reporter aux ouvrages de Noël Coye (2006) et d'Arnaud Hurel (2007).

23. Nakaya donne l'intitulé du cours de l'abbé Breuil en japonais : shinsekki jidai narabi ni kinsekki heiyōki no kaiga chōkoku 新石器時代並びに金石器併用期の絵画彫刻 (Peintures 
rapporte Nakaya de son entretien avec Breuil que ce dernier connaît bien Ōyama Kashiwa 大山柏 (1889-1969) 24, lequel aurait fait une partie de ses études sur le Paléolithique en Europe auprès de Breuil.

Parmi les cours de l'Institut d'ethnologie, Nakaya suit, à la faculté des sciences, ceux d'anthropologie biologique auprès du zoologiste Étienne Rabaud (1868-1956). Il y étudie aussi la géologie du quaternaire et la paléontologie auprès de Léonce Joleaud (1881-1938), président de la Société géologique de France depuis 1928, et il étudie donc "l'ethnographie préhistorique " comme on dit alors, auprès de l'abbé Breuil, à l'Institut de paléontologie humaine. À ces enseignements viennent s'ajouter ceux de Mauss, ce qui laisse penser que Nakaya suit de fait (même si ce n'est jamais explicité) les cours du certificat d'ethnologie de l'Institut.

Le carnet de voyage de Nakaya est souvent imprécis sur les dates de ses rencontres, mais c'est certainement en cette même fin d'année 1929 qu'il va rencontrer le préhistorien André Vayson de Pradenne (1888-1939), qui se lance avec lui dans une grande discussion sur la méthodologie employée par Nakaya, et que ce dernier vient lui présenter (Nakaya 1985 : 76-77). Nakaya exprime dans son carnet une certaine frustration à ne pouvoir suffisamment débattre avec son interlocuteur en raison du niveau de son français. Quoi qu'il en soit, Vayson de Pradenne lui rédige une lettre d'introduction auprès de la Société préhistorique française, dont il va devenir le président en 1930.

et gravures du Néolithique et du Chalcolithique) (Nakaya 1985 : 73-74).

24. Ōyama Kashiwa est une figure de l'archéologie préhistorique japonaise à partir des années 1920. Né dans une riche famille d'aristocrates, militaire de carrière, il part pour l'université de Berlin en janvier 1923, où il étudie l'histoire militaire. Il y découvre en même temps l'archéologie. À son retour, en 1924, il fonde chez lui un laboratoire de Préhistoire (Shizengaku kenkyūshitsu 史前學研究室), lequel deviendra par la suite le laboratoire Ōyama de recherche préhistorique (Ōyama shizengaku kenkyūjo 大山 史前學研究所). En 1929, il fonde la Société préhistorique (Shizen gakkai 史前學会), abritée dans son laboratoire, et lance la revue Shizengaku zasshi 史前學雜誌 (Revue de Préhistoire). Ōyama est l'auteur d'une préface aux " Notions d'archéologie japonaise " de Charles Haguenauer, en 1931 (Haguenauer 1931), auquel il laisse libre accès à son fonds bibliographique. Sur Ōyama, on pourra se reporter à la thèse de Nanta (2004b : 418-427) ainsi qu'à l'ouvrage d'Abe Yoshirō (2004). 


\section{Les années 1930-1931 \\ Les publications en français, la Société préhistorique française, la Société d'anthropologie de Paris, le voyage en Dordogne}

1930 est une année riche pour Nakaya. En décembre 1929, il est d'abord contacté par Louis Joseph Delaporte (1874-1944) ${ }^{25}$. Il demande à Nakaya, pour le compte de la revue Formes, un article grand public présentant l'âge de la pierre de l'archipel japonais. Nakaya, qui ne connaît pas cette revue, demande à Serge Elisséef son avis et ce dernier lui indique qu'elle a une bonne réputation, et s'apparenterait au Japon avec une publication telle que la revue Kaizo 0 改造 (Nakaya 1985 : 78-79). Nakaya signe ainsi, en janvier 1930, son premier article en français, intitulé " Lâge de la pierre au Japon » (Nakaya 1930a). Il y offre une courte histoire de la recherche préhistorique au Japon, et présente des artefacts archéologiques dessinés par ses soins.

Le mois de janvier est également consacré à la préparation d'un autre article, publié cette fois-ci dans l'éphémère (seulement quinze numéros de 1929 à 1931) revue surréaliste, animée notamment par Georges Bataille, Documents $^{26}$. Cet article est intitulé "Figurines néolithiques du Japon " (Nakaya 1930c). Cet article est important car c'est dans ses pages que Nakaya, au-delà d'une simple présentation des figurines préhistoriques japonaises, les $\operatorname{dog} \bar{u}$ 土偶, expose ses intentions de recherche à venir :

"faire une étude sur ces figurines et éclaircir leur évolution typologique, leur tendance à la dégénérescence, les relations entre les objets trouvés ensemble, leurs propriétés spéciales dans chaque région déterminée, etc. Je désirerais fournir un exemple de mes études sur le néolithique au Japon pour y déterminer les courants d'une culture, les relations entre les courants de la même culture, son expansion ainsi que l'influence des autres cultures sur elle. » (Nakaya 1930c : 32)

Nakaya a beau venir du Japon où l'archéologie n'est pas encore une discipline imposante, il est au même diapason que bien de ses collègues

25. Louis Joseph Delaporte, fondateur, en 1922, de la Société des études hittites et asianiques, et professeur à l'Institut catholique de Paris.

26. Sur Documents, et plus généralement sur le Collège de sociologie, voir l'ouvrage de Denis Hollier (1995). 


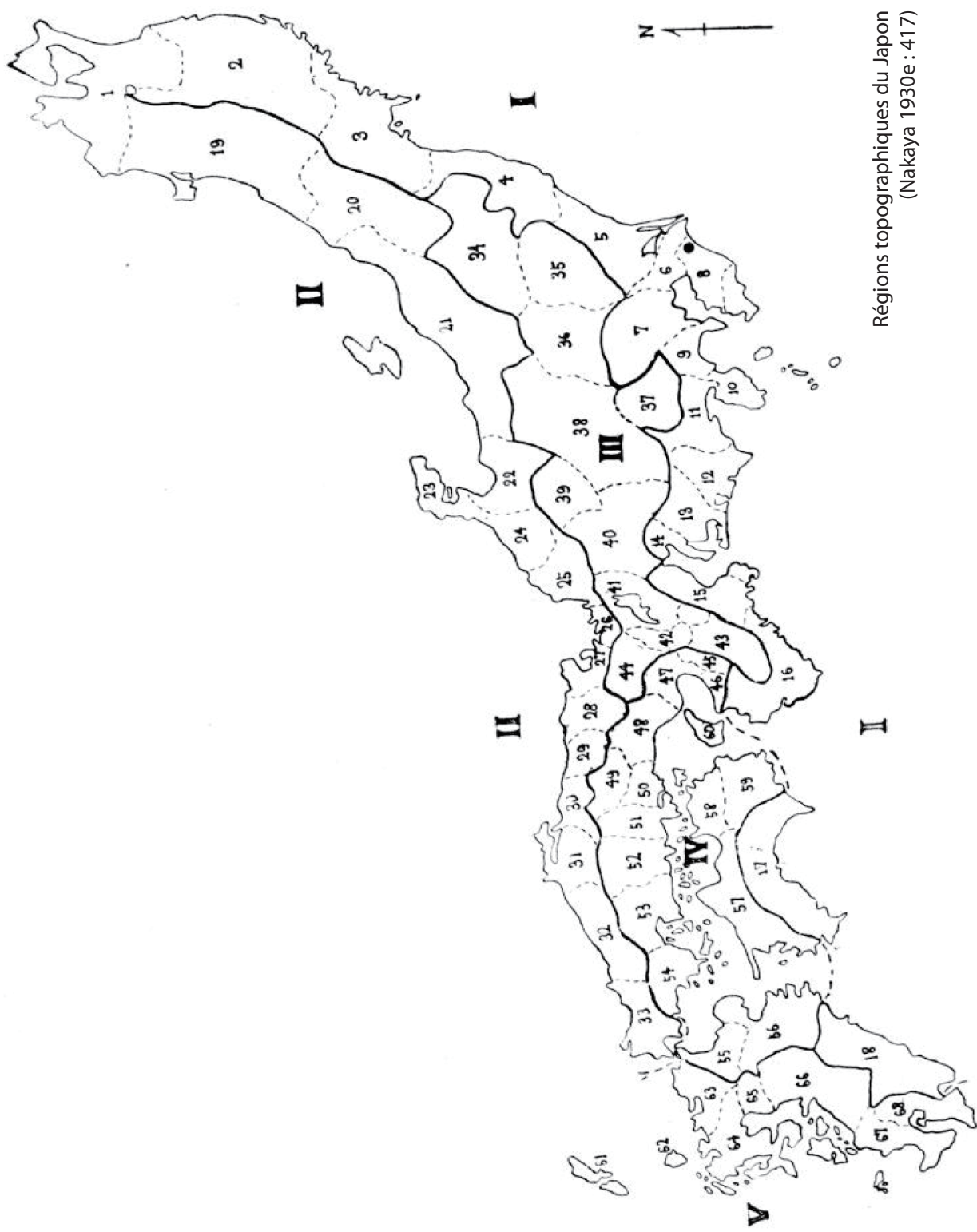


européens, cherchant à élaborer une histoire des cultures archéologiques et de leur aire d'appartenance, c'est-à-dire en fait une histoire des peuples.

À l'insistance de Vayson de Pradenne, Nakaya fait donc son entrée à la Société préhistorique française, où il expose ses travaux le 26 juin 1930. Suivent alors deux articles qui constituent le noyau dur de l'activité de recherche de Nakaya en France : "Introduction à l'étude des figurines de l'âge de la pierre au Japon " (Nakaya 1930d) et "Introduction à l'étude des figurines néolithiques au Japon " (Nakaya 1930e). C'est dans ces deux articles que Nakaya expose en français la méthode combinée dont il débattait en 1929 avec Rivet dans le bureau de ce dernier : carte de « distribution quantitative " des figurines selon les sites, croisée avec les résultats obtenus par la même méthode dans son étude de 1927 sur les poteries à bec verseur $^{27}$ (Nakaya 1930d : 22).

Nakaya rédigera également deux articles pour la Revue des Arts asiatiques. Une publication en 1930, intitulé " Contribution à l'étude de la civilisation néolithique au Japon " (Nakaya 1930b), qu'il traduira ultérieurement en japonais pour la revue Kökogaku de son ami Morimoto (Nakaya 1936) ${ }^{28}$. C'est une contribution importante car il s'agit d'un des premiers travaux de l'histoire de l'archéologie japonaise à vraiment essayer de mettre de l'ordre spatialement et chronologiquement entre les cultures matérielles de l'archipel représentées par les deux types de poterie que sont Yayoi et Jōmon. Dans la version traduite du français vers le japonais des travaux de Nakaya, on remarque que le terme de "civilisation ", qu'il emploie seul ou dans l'expression de "domaine de civilisation ", est traduit en japonais par le terme bunka 文化, qui signifie " culture ", et correspond en archéologie au terme allemand de Kultur. Cela ne fait que souligner que s'il utilise bien, en France, le terme de "civilisation ", comme il est d'usage, au Japon en revanche, c'est la terminologie scandinave ou allemande qui détermine le choix du terme : bunka plutôt que bunmei 文明 (civilisation).

En 1931, il publie, encore dans la Revue des Arts asiatiques, un article intitulé "L'influence des civilisations continentales sur l'âge de pierre au Japon » (Nakaya 1931). Cette contribution fait l'objet d'une traduction, en

27. Nakaya emploie le terme de "vase à bec" (Nakaya 1930d).

28. Voir les fac-similés dans Saitō (1972 : 27-47). 


\begin{tabular}{|c|c|c|c|c|c|c|c|}
\hline REG GIONS & $\mathrm{N} \cdots$ & DISTRICTS & SITES & RE GIONS & $\mathrm{N} 0 *$ & DISTRICTS & SITES \\
\hline \multirow{10}{*}{ I } & 1 & Mutsu & 36 & & & & \\
\hline & 2 & Rikuchu & 46 & \multirow{8}{*}{ III } & 34 & Iwashiro & 23 \\
\hline & 3 & Rikuzen & 17 & & 35 & Shimozuké & 13 \\
\hline & 4 & Swaki & 16 & & 36 & Kozuké & 9 \\
\hline & 5 & Hidachi & 33 & & 37 & Kabi & 12 \\
\hline & 6 & Shimofusa & 41 & & 38 & Shinano & 70 \\
\hline & 7 & Musashi & 37 & & 39 & Hida & 1 \\
\hline & \multirow{2}{*}{$\begin{array}{r}8 \\
9 \\
13\end{array}$} & \multirow{3}{*}{$\begin{array}{l}\text { Sagami } \\
\text { Mikawa }\end{array}$} & 5 & & 40 & Mino & 1 \\
\hline & & & \multirow{2}{*}{5} & & $\frac{43}{-12}$ & Yamato & \\
\hline & & & & & 51 & Bittsu & 2 \\
\hline \multirow{4}{*}{ II } & $\begin{array}{l}19 \\
20\end{array}$ & $\begin{array}{l}\text { Ugo } \\
\text { Uzen }\end{array}$ & $\begin{array}{r}23 \\
7\end{array}$ & & & Toshima & 4 \\
\hline & 21 & $\begin{array}{l}\text { Uzen } \\
\text { Echigo }\end{array}$ & 8 & & & $\begin{array}{l}\text { Kosshi } \\
\text { Ishikari }\end{array}$ & 1 \\
\hline & 22 & Etchì & 5 & & & Iburi & 4 \\
\hline & & Echizen & 1 & & 28 & TOTAL & 429 \\
\hline
\end{tabular}

Tableau 1. - Nombre des sites de figurines par district.

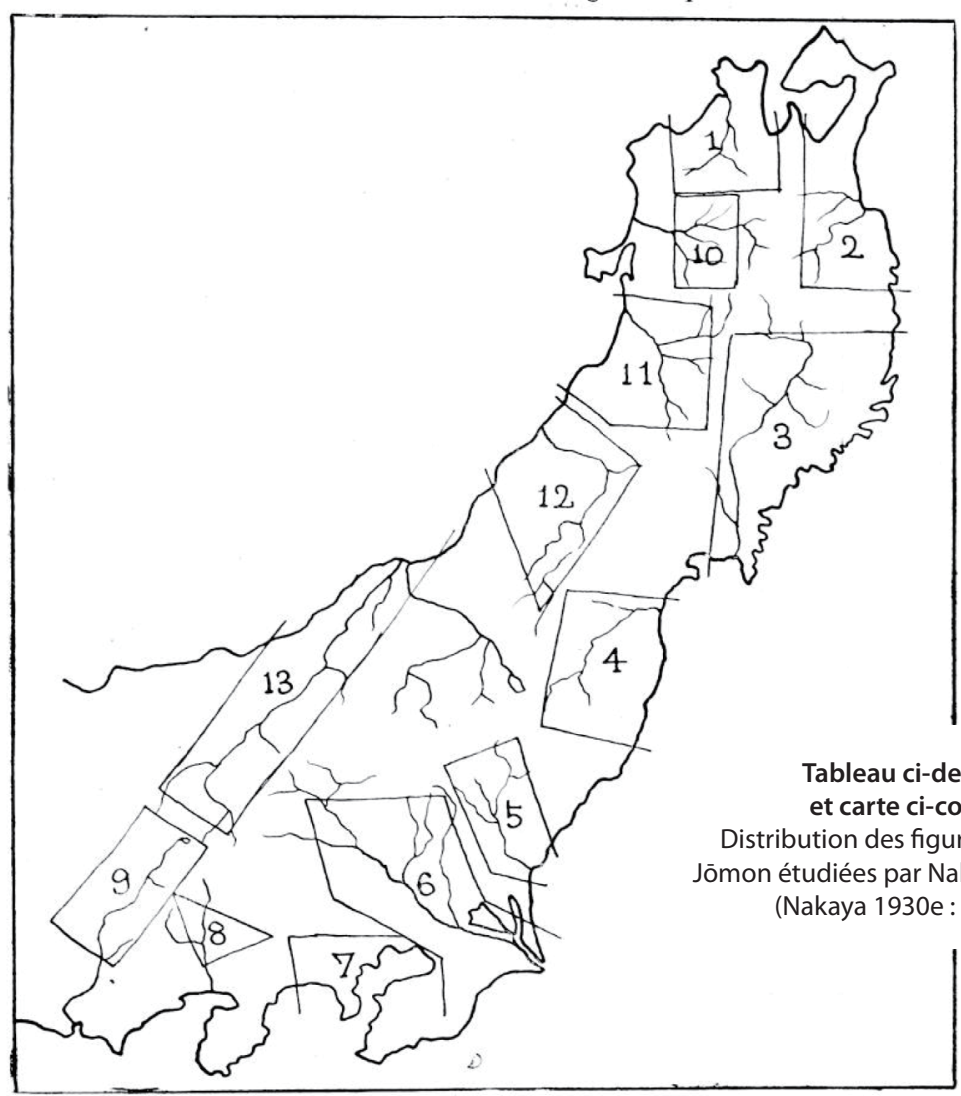

Carte 2. -- Zones de distribution de figurines. 


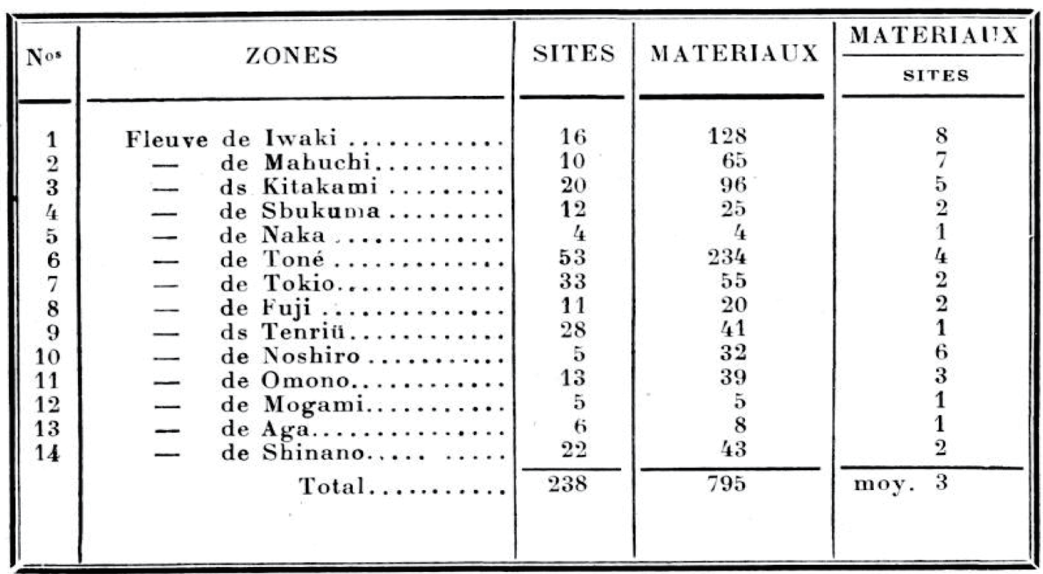

Tableau 2. - Zones de distribution et nombre de moyenne des figurines.

\begin{tabular}{|c|c|c|c|c|c|c|}
\hline \multicolumn{2}{|r|}{ LOCALITE } & \multicolumn{4}{|c|}{ TYPE } & \multirow[b]{2}{*}{$\mathrm{T}$} \\
\hline ZONES & TOPOGRANHIE: & A & B & C & $\mathrm{D}$ & \\
\hline $\begin{array}{l}1 \\
2 \\
4 \\
3 \\
5 \\
6 \\
7 \\
8\end{array}$ & $\begin{array}{l}\text { Baie de Tokio } \\
\text { - Kasumigaura } \\
\text { Fleuve de Kitakami } \\
\text { Bord de Rikuzen } \\
\text { Fleuve de Mabuchi } \\
\text { - de Iwaki } \\
\text { - de Nosbiro } \\
\text { - de Omono }\end{array}$ & $\begin{array}{r}31 \\
12 \\
3 \\
1 \\
- \\
- \\
-\end{array}$ & $\begin{array}{r}3 \\
45 \\
23 \\
8 \\
6 \\
15 \\
9 \\
6\end{array}$ & $\begin{array}{r}1 \\
1 \\
8 \\
15 \\
51 \\
38 \\
22 \\
31\end{array}$ & $\begin{array}{r}- \\
5 \\
6 \\
22 \\
5 \\
9 \\
12\end{array}$ & $\begin{array}{l}35 \\
58 \\
39 \\
30 \\
79 \\
58 \\
40 \\
4 y\end{array}$ \\
\hline & $\mathrm{T}$ & 47 & 115 & 167 & j9 & 388 \\
\hline
\end{tabular}

Ta!jla:1 3. - Les distributions quantitatives des vases á becs. 


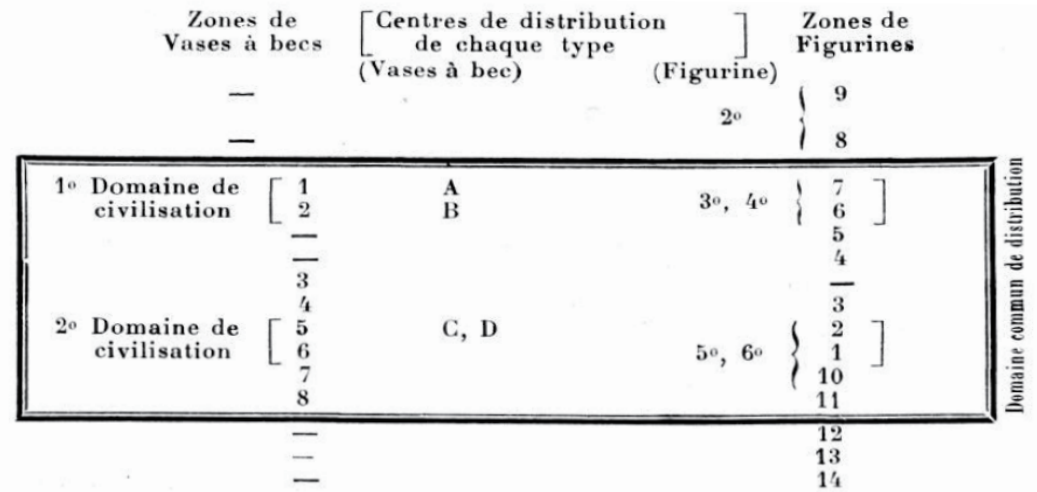

Tableau 4. - Relations entre les zones de distribution de vases avec becs et de figurines. 
1934, dans la revue Kökogaku (Nakaya 1934) ${ }^{29}$. Ces deux derniers articles apportent des éléments de synthèses supplémentaires à la genèse de ce que Nakaya appelle donc le " néolithique » du Japon, au travers de la construction de "domaines de distribution " et de "domaines de civilisation ", matérialisés au travers d'aspects " autochtones " ainsi qu’allogènes.

On peut ainsi dire que Nakaya est, dans les deux premières années de la décennie 1930, non seulement le principal vecteur de la connaissance sur la Préhistoire de l'archipel en France (et en Europe), mais également un des tout premiers archéologues à proposer - outil scientifique à la main - des perspectives de définition chronologique et spatiale sur ce qu'il appelle la " civilisation néolithique » de l'archipel, à savoir, pour lui, la culture à poterie de type Jōmon (poterie à motif en impression de corde).

\section{L'entrée à la Société d'anthropologie de Paris et le voyage en Dordogne}

En mars 1930, Nakaya est contacté par le président de la Société d'anthropologie de Paris, le docteur Victor Bugiel (Nakaya 1985 : 80-81). Invité un soir chez ce dernier à dîner, Nakaya se fait assaillir de questions : sur les raisons pour lesquelles les anthropologues japonais publient en anglais, en allemand, mais jamais en français ; pour savoir si les étudiants japonais en médecine se consacrent beaucoup à l'anthropologie ; sur l'épaisseur des murs des maisons ; sur comment la vie est possible dans des maisons où il n'y a pas de système de chauffage en hiver, etc. Pour répondre aux questions les plus "scientifiques " de Bugiel, Nakaya rédigera un article dans les colonnes du Bulletin et mémoires de la Société d'anthropologie de Paris. Cette contribution est intitulée "Sur les études anthropologiques actuelles au Japon " (Nakaya 1930f) ${ }^{30}$. Nakaya devient ainsi membre de la Société, en 1931, sur recommandation de son président, de son secrétaire général R. Anthony et d'Adrien de Mortillet (1853-1931), président d'honneur de la Société préhistorique française et qui mourra la même année.

Enfin, son voyage, à l'été 1931, en Dordogne, en compagnie de nombreuses personnes de l'Institut, semble avoir été marquant puisqu'il y

29. Voir le fac-similé dans Saitō (1972 : 14-27).

30. Article signé Nakaya Jinjiro (prénom mal orthographié par la revue). 
consacre près de dix pages dans son carnet (Nakaya 1985 : 103-112). Parmi les voyageurs on compte Joleaud, Rabaud, mais aussi le couple Montandon (1879-1944). Contrairement à ce que nous aurions pu penser, Nakaya ne développe pas au sujet de George Montandon ${ }^{31}$, qui s'intéresse pourtant au Japon, particulièrement aux Aïnous, depuis son voyage en Sibérie entre 1919 et 1921 (Montandon 1921) ${ }^{32}$. En route, tous s'arrêtent au laboratoire de Léon Henri-Martin (1864-1936), l'un des fondateurs de la Société préhistorique française, plusieurs décennies plus tôt. Henri-Martin habite sur son lieu de travail, à La Quina, riche gisement archéologique qu'il étudie depuis plus de vingt ans alors. Commence ainsi une excursion de sites, surtout des grottes, dont certains ont fait l'histoire de la Préhistoire : La Quina $^{33}$, mais aussi la grotte ornée du Roc de Sers, La Micoque, la grotte de Font de Gaume, Laugerie Haute et Laugerie Basse, l'abri du Cap Blanc, le Moustier. C'est aussi une des caractéristiques de la présence de Nakaya en France : il a le sentiment d'être présent au moment où un premier profond travail de définition, fondamental, de la Préhistoire est en train de s'achever.

31. Nakaya rapporte toutefois que lors de la réunion de mars 1930 de la Société, Montandon fait une conférence très générale d'ethnographie comparée autour des baguettes gravées des Aïnous (les ikupasuiイクパスイ) et leur « équivalent » du Paléolithique. Nakaya rapporte que le contenu de la conférence devait s'avérer finalement plutôt maigre (Nakaya 1985 : 87).

32. En 1929, Montandon est déjà l'auteur de plusieurs articles dans lesquels il est question des Aïnous et des Japonais, dont deux publiés dans la revue L'Anthropologie sous des titres évocateurs : "Crâniologie paléosibérienne : néolithiques, mongoloïdes, tchouktchi, eskimo, aléoutes, kamtchadales, aïnou, ghiliak, negroïdes du nord " (Montandon 1926) ; "Aïnous, Japonais, Bouriates " (Montandon 1927a). Un ouvrage sort ensuite, intitulé Au Pays des Aïnous : exploration anthropologique (Montandon 1927b).

33. Nakaya rapporte que lui et ses compagnons vont fouiller une matinée sur les niveaux aurignaciens du gisement, et auront le droit d'emporter avec eux les silex taillés découverts (Nakaya 1985 : 105). 


\section{En guise de conclusion Nakaya Jiujirō, à la croisée des chemins}

Les travaux de Nakaya s'inscrivent ainsi, nous le voyons bien, fort à propos dans les préoccupations de l'Europe de son temps. Ils s'inscrivent également fort bien au sein du laboratoire d'anthropologie de l'université impériale de Tokyo, qui a formé à cette époque une génération d'archéologues préhistoriens marqués par les méthodes de l'archéologie et de l'anthropologie. Nakaya, ou encore Yamanouchi Sugao 山内清男 (1902-1970), connu après-guerre pour ses travaux sur le Jōmon, sont de cette génération. L’approche archéologique de Nakaya reste originale au Japon à son époque : typologie de la poterie à bec verseur de type Jōmon, typologie des figurines $\operatorname{dog} \bar{u}$, et enfin appréhension de ces artefacts dans l'espace afin de déterminer - ce qu'il désigne, en français -, des "domaines de civilisation".

De la même manière que la " méthode cartographique " de Nakaya s'inscrit dans cette démarche de spatialisation, nous pouvons dire que le souci dont il témoigne de vouloir désigner les "influences de la culture continentale ", notamment dans ses derniers articles, viennent également rejoindre la question du métissage traitée par l'anthropométrie. Si Matsumura peut être, en quelque sorte, qualifié d'archéologue des " domaines de morphotype ", Nakaya serait assez justement compris comme un archéologue des "domaines de civilisation ", au sens de cultures archéologiques.

Les travaux de Nakaya, au-delà de la question de leur validité qui ne nous intéresse que peu ici, sont aussi révélateurs de certains blocages de l'archéologie japonaise en général à cette époque. Il désigne la période de la poterie de type Jōmon, en français, puis finalement en japonais dans ses derniers travaux, comme relevant du Néolithique de l'archipel. Il parle, pour la poterie de type Yayoi, qui lui succède sur le plan chronologique, d'énéolithique, ou de protohistoire si l'association avec des objets en bronze ou en fer est constatée (Nakaya 1930b : 151). Il élimine d'emblée le terme de paléolithique, comme le laisse comprendre d'ailleurs son entretien avec Boule, dans la mesure où tous les chercheurs japonais de son époque considèrent très improbable la présence de l'homme fossile au Japon. "Néolithique " est donc un terme utilisé, chez Nakaya, " contre le Paléolithique », non pas parce qu'il y aurait eu une « révolution 
néolithique ${ }^{34}$ ", mais parce que le "Paléolithique " est encore impensable et impensé pour le Japon. C'est aussi sans doute dans ce sens qu'il faut interpréter les questions sur le Mésolithique d'Europe, posées par Nakaya à Boule : dans le contexte épistémologique du Japon de Nakaya, l'idée de Mésolithique, temps situé juste " en dessous " des couches stratigraphiques du Néolithique, constituerait une alternative possible à celle de Paléolithique. Les typologies de Nakaya, techniquement tout à fait méritantes au regard des exigences de son temps, font aussi les frais de cet obstacle gênant pour penser un temps plus profond. Nakaya ne parvient jamais, malgré la relative richesse du matériel qu'il utilise, à rendre compte de la "profondeur temporelle " - ou du "domaine de temps " pour fabriquer un mot à la manière de Nakaya -, dans laquelle s'inscrit la poterie de type Jōmon. Les typo-chronologies de Nakaya sont toutes, à notre connaissance, le plus souvent arrimées à une échelle de temps relativement courte, articulée à la protohistoire.

La typologie constitue un outil servant les objets mesurés par la "méthode quantitative ", laquelle rend compte de la densité d'un type d'un site à l'autre. La méthode cartographique vient, dans un second temps, donner un visage, un sens de civilisation (culture) à la disparité de ces densités. On comprend la nécessité des inventaires - exhaustifs pour leur époque - élaborés par Nakaya. Venir étudier auprès de Paul Rivet, dont le souci méticuleux pour les fiches d'objet en vue de l'étude des "Civilisations matérielles " n'est pas un secret, constituait un choix somme toute naturel pour Nakaya.

Aujourd'hui, à l'heure où les archéologies européennes peuvent s'enorgueillir de certains programmes coopératifs ${ }^{35}$, il n'existe toujours pas de

34. L'idée de " révolution néolithique " de Childe est peut-être arrivée aux oreilles de Nakaya, compte tenu de l'époque, mais il est impossible d'en percevoir la trace dans ses travaux. On n'en trouve pas non plus de trace probante dans ses échanges avec Morimoto durant son séjour à Paris. Tout cela laisse penser que l'impact de Childe est demeuré dans un premier temps fort limité. En outre, on pourra aussi ajouter que lorsque Morimoto, en 1933, parle des premières sociétés rizicoles de l'archipel, c'est dans ses propres termes et il est difficile de vraiment relier (terminologie, argumentation, etc.) sa pensée à celle de Childe.

35. On peut citer ici les fouilles du mont Lassois, organisées en plusieurs équipes, dont une équipe suisse, une équipe autrichienne et une équipe franco-allemande. Les fouilles 
vigoureuse collaboration franco-japonaise (ou entre les pays d'Europe continentale et le Japon) dans le domaine de l'archéologie. Les archéologies d'Europe du Nord, de l'Ouest et du Japon sont pourtant désormais assez anciennes pour avoir eu le temps de se rencontrer, et il est donc à regretter que les échanges ne se soient toujours pas systématisés. Le paléolithicien Kagawa Mitsuo 賀川光男 a pu écrire qu’il avait été nécessaire d'attendre les années 1960 pour qu'une nouvelle fenêtre s'ouvre depuis le Japon, sur les activités archéologiques françaises en France (Kagawa 1985). Nakaya est donc en lui-même original ne serait-ce que par son ouverture à l'archéologie européenne d'une part, et, d'autre part, du fait de la force de son engagement scientifique auprès des préhistoriens français durant son séjour : son activité montre qu'il n'était pas là pour faire du " tourisme scientifique ".

Nakaya Jiujirō était sans doute appelé à devenir un nom important de l'archéologie japonaise, comme le démontre la réédition en 1943 de son manuel de 1929 et donc l'intérêt pour celui-ci. En outre, compte tenu de son intégration dans le monde de la recherche de l'époque en France, on pouvait voir sous un jour très prometteur l'avenir de futures collaborations archéologiques entre la France et le Japon. Quand il rentre au Japon, Nakaya est d'ailleurs nommé Délégué pour le Japon par la Société préhistorique française $e^{36}$. Par ses écrits en français et son activité au sein des sociétés de l'époque, il est la première source faisant appel à un discours et à des méthodes rigoureusement construits par laquelle les archéologues français peuvent s'ouvrir au Japon.

Nakaya tombe malade, quitte la France en 1932, pour ensuite décéder à l'âge de 34 ans quelques années plus tard. Il en va de même pour Morimoto que nous avons évoqué. Ces deux préhistoriens laissent ainsi un vide dans le sens où, d'une part, ils ne seront pas en mesure de construire de liens forts entre la France et le Japon sur le plan de la recherche archéologique et, d'autre part, nous ne pourrons pas savoir quel aurait été le visage de l'archéologie qu'ils auraient ensuite développée au Japon, du fait de leur expérience à Paris.

sur les oppida de l'âge du fer, comme celles du mont Beuvray, constituent un autre exemple de collaboration internationale.

36. Cf. séance du 23 juin 1932, Bulletin de la Société préhistorique française (1932). 


\section{Bibliographie}

\begin{abstract}
ABE Yoshirō 阿部芳郎, 2004
Ushinawareta shizengaku. Kōshaku

Ōyama Kashiwa to Nihon kōkogaku

失われた史前学 一一公爵大山柏と日本

考古学 (Une Préhistoire perdue. Le duc Ōyama Kashiwa et l'archéologie japonaise), Tokyo, Iwanami shoten 岩波書店.
\end{abstract}

AKUTAGAWA Ryūnosuke, 1971 Akutagawa Ryūnosuke zenshū 芥川龍之 介全集 (Fuvres complètes d'Akutagawa Ryūnosuke), 9 vol., Tokyo, Chikuma shobō 筑摩書房.

\section{Bulletin de la Société préhistorique} française, 1932

«Séance du 23 juin 1932 », $29: 258$.

\section{CHILDE Vere Gordon, 1923}

How Labour Governs, Melbourne, Melbourne University Press.

\section{CHILDE Vere Gordon, 1925}

The Dawn of European Civilization, Londres, Paul Kegan.

\section{COYE Noël, 2000}

La Préhistoire en paroles et en actes. Méthodes et enjeux de la pratique archéologiques (1830-1950), Paris, L'Harmattan.

\section{COYE Noël, 2006}

Sur les chemins de la Préhistoire. L'abbé Breuil, du Périgord à l'Afrique du Sud, Paris, Somogy.
COYE Noël \& HUREL Arnaud (dir.), 2011

Dans l'épaisseur du temps, Paris, Publications scientifiques du Muséum.

DÉCHELETTE Joseph, 1908-1914 Manuel d'archéologie préhistorique et celtique, en deux tomes: t. I. Archéologie préhistorique; t. II. Archéologie protohistorique ou celtique, subdivisé en 3 volumes : vol. 1 L'âge du bronze; vol. 2 Premier âge du fer ou époque de Hallstatt ; vol. 3 Second âge du fer ou époque de la Tène, Paris, Picard et Fils.

\section{HAGUENAUER Charles, 1931}

" Notions d'archéologie japonaise », Bulletin de la Maison franco-japonaise, t. III, 1-2:1-78.

\section{HOLLIER Denis, 1995}

Le Collège de sociologie 1937-1939, Paris, Gallimard (édition revue et augmentée).

HUREL Arnaud, 2007

La France préhistorienne de 1789 à 1941, Paris, CNRS Éditions.

KOBAYASHI Yukio 小林行雄 (dir.), 1971 Ronshū Nihon bunka no kigen 1 論集 日本文化の起源 1 (Recueil de textes. Les origines de la culture japonaise, vol. 1), Tokyo, Heibonsha 平凡社.

KROEBER Alfred L. \& COLLIER Donald (edited by Patrick H. CARMICHAEL), 1998

The Archaeology and Pottery of Nazca, 
Peru: Alfred Kroeber's 1926 Expedition, Walnut Creek, Altamira Press.

\section{LAURIÈRE Christine, 2008}

Paul Rivet : le savant et le politique, Paris, Muséum national d'histoire naturelle.

\section{LEHOËRFF Anne, 2009}

« Les paradoxes de la Protohistoire française ", Annales. Histoire, Sciences sociales, 64e année, 5 : 1107-1133.

\section{KAGAWA Mitsuo 賀川光男, 1985}

« Nakaya Jiujirō no Pari ryūgaku jidai » 中谷治宇二郎のパリの留学時代 (Nakaya Jiujirō à l'époque de son voyage d'études à Paris), in NAKAYA 1985, p. 165-183.

MORIMOTO Rokuji 森本六爾, 1929 Nihon seidōki jidai chimei-hyō 日本青銅器 時代地名表 (Inventaire des gisements de l'âge du bronze au Japon), Tokyo, Oka shoin 岡書院.

\section{MORIMOTO Rokuji, 1933}

Nihon genshi nōgyō 日本原始農業 (Une agriculture primitive au Japon), Tokyo, Tōkyō Kōkogakkai 東京考古學會.

\section{MONTANDON George, 1921}

"Mission en Sibérie ", Revue internationale de la Croix-Rouge et Bulletin international des Sociétés de la Croix-Rouge, 3 : 1197-1232.

\section{MONTANDON George, 1926}

«Craniologie paléosibérienne: néolithiques, mongoloïdes, tchouktchi, eskimo, aléoutes, kamtchadales, aïnou, ghiliak, negroïdes du nord ", L'Anthropologie, 36 : s. 209-296, 447-542.

MONTANDON George, 1927a «Aïnous, Japonais, Bouriates », L'Anthropologie, 37 : 97-124.

\section{MONTANDON George, 1927b}

Au Pays des Aïnous : exploration anthropologique, Paris, Masson \& Cie.

MORTILLET (de) Gabriel, [1883] 1885 Le préhistorique. Antiquité de l'homme, Paris, Reinwald.

NAKAYA Jiujirō 中谷治宇二郎, 1927 Chūkō doki no bunrui to sono chiriteki bunpu 注口土器ノ分類と其ノ地理的 分布 (Classification et répartition géographique de la poterie chūkō), Tokyo, Oka shoin 岡書院.

\section{NAKAYA Jiujirō,1929}

Nihon sekki jidai teiyō 日本石器時代提要 (Précis sur l'âge de la pierre au Japon), Tokyo, Oka shoin.

NAKAYA Jiujirō [Jiujiro], 1930a "L'âge de la pierre au Japon », Formes, IV:9-11.

NAKAYA Jiujirō [Jiujiro], 1930b «Contribution à l'étude de la civilisation néolithique au Japon », Revue des Arts asiatiques, VI (3) : 151-167.

NAKAYA Jiujirō [Jiujiro], 1930c "Figurines néolithiques du Japon », in Documents, $1: 25-32$. 
NAKAYA Jiujirō [Jiujiro], 1930d «Introduction à l'étude des figurines de I'âge de la pierre au Japon », Jahrbuch Für Prähistorische \& Ethnographische Kunst, 6 : 19-30.

NAKAYA Jiujirō [Jiujiro], 1930e « Introduction à l'étude des figurines néolithiques au Japon », Bulletin de la Société préhistorique française, 27 : 416-426.

NAKAYA Jiujirō [Jinjiro], $1930 f$ «Sur les études anthropologiques actuelles au Japon », Bulletin et mémoires de la Société d'anthropologie de Paris, $1(1-1-3): 8-12$.

\section{NAKAYA Jiujirō,1930g}

Nihon sekkijidai bunken mokuroku 日本石器時代文獻目錄 (Inventaire des sources sur l'âge de la pierre au Japon), Tokyo, Oka shoin.

\section{NAKAYA Jiujirō [Jiujiro], 1931}

«L'Influence des Civilisations continentales sur l'âge de pierre au Japon », Revue des Arts asiatiques, 7 (3) : 141-155.

\section{NAKAYA Jiujirō,1934}

« Nihon sekki jidai ni okeru tairiku bunka no eikyō »日本石器時代における 大陸文化の影響 (L'Influence des cultures continentales sur l'âge de la pierre au Japon), Kōkogaku 考古學, 5 (4)； fac similé in SAITŌ 1972 : 14-27.

\section{NAKAYA Jiujirō,1935}

Nihon senshigaku joshi 日本先史學序史 (Histoire préliminaire à la recherche en
Préhistoire au Japon), Tokyo, Iwanami shoten 岩波書店.

\section{NAKAYA Jiujirō,1936}

« Nihon shinsekki bunka no ichikōkyū : Toku ni bunpu-ken to bunka-ken ni tsuite »日本新石器文化の一考究

—— 特に分布圈と文化圈に就て

(Contribution à l'étude du Néolithique du Japon : sur les domaines de distribution et les domaines de culture), Kōkogaku, 7 (1-2).

\section{NAKAYA Jiujirō, 1943 [1929]}

Kōtei Nihon sekki jidai teiyō 校訂日本石器 時代提要 (Précis sur l'âge de la pierre au Japon, édition revue), Tokyo, Oka shoin.

\section{NAKAYA Jiujirō,1985}

Kōkogaku kenkyū e no tabi. Pari no shuki 考古学研究への旅 — パリの手記 (Un voyage pour l'archéologie. Carnet de Paris), Tokyo, Rokkō shuppan 六甲出版.

\section{NAKAYA Ukichirō 中谷宇吉郎， [1988] 2011 \\ Nakaya Ukichirō zuihitsu-shū 中谷宇吉郎随筆集 (Recueils de notes de Nakaya Ukichirō), Tokyo, Iwanami shoten 岩波書店.}

NANTA Arnaud, 2004a «Débat autour des fouilles archéologiques à Ōsaka, 1917-1920», Ebisu. Études japonaises, 32 : 25-63

NANTA Arnaud, 2004b

Débats sur les origines du peuplement de l'archipel japonais dans l'anthropologie et l'archéologie (décennie 1870 - décennie 
1990), thèse de doctorat, université

Paris 7 - GHSS.

\section{NESPOULOUS Laurent, 2003}

« Des empereurs et des tombes, Une archéologie de l'archéologie protohistorique japonaise à l'époque d'Edo », Ebisu. Études japonaises, $30: 87-122$.

\section{NESPOULOUS Laurent, 2004} « Mémoire, symbole, tradition et archéologie impériale. Évolution de l'archéologie des tertres protohistoriques, de la Restauration impériale à la fin des années 1930 », Ebisu. Études japonaises, 32 : 3-24.

\section{NESPOULOUS Laurent, 2012}

«Memories from Beyond the Past. Grasping Prehistoric Times in Japan: The Birth and Evolution of an "Archaeological Consciousness" (17th to 20th Centuries) », in Rosa CAROLI \& Pierre-François SOUYRI (dir.), History at Stake in East Asia, Venise, Cafoscari, p. 107-118.

\section{REYNAUD-PALIGOT Carole, 2001}

«Paul Rivet : contradictions et ambiguïtés d'un intellectuel antiraciste dans les années trente », in Claude LIAUZU \& Marie-Claire HOOKDEMARLE (dir.), Transmettre les passés: nazisme, Vichy, conflits coloniaux. Les responsabilités des universités, Paris, Syllepse, p. 160-173.

\section{RIVET Paul, 1929}

«L'étude des civilisations matérielles: ethnographie, archéologie, préhistoire », Documents, 3 : 130-134.

\section{SAITŌ Tadashi 斎藤忠 (dir.), 1972}

Nihon kōkogaku senshū 24 日本考古学 選集24 (Recueil d'articles choisis sur l'archéologie japonaise, vol. 24), Tokyo, Tsukiji shokan 築地書館.

\section{SAPIRO Gisèle, 2009}

L'espace intellectuel en Europe.

De la formation des États-nations

à la mondialisation, $X I x^{e}-X X l^{e}$ siècle, Paris, La Découverte.

\section{SCHLANGER Nathan, 2013}

" Hero of One Day. Jules Reboux at the Crucible of Prehistory in 1860s Paris ", Complutum, 24 (2) : 73-88.

\section{SCHNAPP Alain, 1982}

"Archéologie et tradition académique en Europe aux xVIII ${ }^{\mathrm{e}}$ et $\mathrm{xIx}$ siècles ", Annales ESC, 37 (5-6) : 760-777.

\section{TŌKYŌ TEIKOKU DAIGAKU}

\section{東京帝國大學, 1928}

Nihon sekki jidai ibutsu hakken chimeihyō 日本石器時代遺物發見地名表 (Inventaire des gisements archéologiques de l'âge de la pierre au Japon), Tokyo, Oka shoin 岡書院. 